\title{
Article \\ Structural Design and Parameter Optimization of Bionic Exhaust Tailpipe of Tractors
}

\author{
Zhenhua Hou, Qigan Wang, Shiqiang Zhang, Tengfei Si, Tiange Li and Zhijun Zhang *(1) \\ Key Laboratory of CNC Equipment Reliability (Ministry of Education), School of Mechanical and Aerospace \\ Engineering, Jilin University, Changchun 130022, China; houzh19@mails.jlu.edu.cn (Z.H.); \\ wangqg21@mails.jlu.edu.cn (Q.W.); zsq20@mails.jlu.edu.cn (S.Z.); sitf20@mails.jlu.edu.cn (T.S.); \\ litg20@mails.jlu.edu.cn (T.L.) \\ * Correspondence: zhijunzhang@jlu.edu.cn
}

\begin{abstract}
The exhaust tailpipe of a certain type of tractor was improved from the perspective of bionics, and bionic triangular convex texture was added to the inner surface of the exhaust tailpipe. The bionic tailpipe was proposed to improve noise reduction performance without changing the overall size parameters of the prototype tailpipe. Acoustics simulation software was used to predict the aeroacoustics noise and transmission loss of the exhaust tailpipe. Bionic exhaust tailpipes with triangular textures of different numbers of circumferential columns, height, and top angles were analyzed to study the noise reduction performance. The results showed that the proposed bionic exhaust tailpipes with triangular convex textures reduced the total sound pressure level and improved the transmission loss of the prototype exhaust tailpipe. To increase the transmission loss, a genetic algorithms (GA) optimized back-propagation neural network (BP) was used to optimize the bionic triangular convex texture parameters. By studying the aerodynamic noise reduction mechanism of bionic tailpipes, the research suggested that a secondary vortex appeared near the bionic texture and reduced aerodynamic drag and aeroacoustics noise. In addition, the sound pressure level amplitude nephogram, velocity vector nephogram, and velocity amplitude nephogram of the exhaust tailpipes were analyzed to study the vibration noise reduction mechanism of the bionic tailpipes. Then, the noise reduction performance was experimentally evaluated. The experimental results of the bionics exhaust tailpipes with triangular convex textures were analyzed and compared to that of the prototype tailpipe. The results demonstrated that the bionic exhaust tailpipes were able to attenuate noise.
\end{abstract}

Keywords: exhaust tailpipe; aerodynamic noise; vibration noise; bionic triangular texture

\section{Introduction}

The exhaust noise generated by tractors is a problem that puzzles people. Experts and Publisher's Note: MDPI stays neutral with regard to jurisdictional claims in published maps and institutional affiliations.

Copyright: (ㅇ 2022 by the authors. Licensee MDPI, Basel, Switzerland. This article is an open access article distributed under the terms and conditions of the Creative Commons Attribution (CC BY) license (https:// creativecommons.org/licenses/by/ $4.0 /)$. scholars have been paying attention to the goal of reducing exhaust noise. Mufflers are widely used to reduce intake and exhaust noise.

In recent years, researchers proposed various methods to improve the noise reduction performance of mufflers. Fu et al. used a simulated method and experimental test to obtain the transmission loss and analyzed the influence of several main parameters of mufflers on transmission loss $[1,2]$. Cambonie et al. studied curved quarter-wave resonators to solve the problem of large spaces occupied by straight cavity resonators [3]. Xue et al. applied U-shaped bellows to a resistant muffler, and the noise reduction performance was significantly improved in the cutoff frequency range [4]. Segin et al. used the finite element analysis method to optimize the acoustic performance of a multi-chamber reactive silencer with baffles [5]. Sagar et al. designed an H-type connecting fork muffler based on an H-Q tube. This muffler fundamentally changed the acoustic impedance at the sound source [6]. Zhu et al. studied a new type of semiactive muffler based on an H-Q pipe to control the 
low-frequency noise of the exhaust pipe [7]. Mimani et al. analyzed the transmission loss performance of the rectangular expansion chamber with a single inlet and single outlet and single inlet and double outlet by using the three-dimensional semi-analytic formula based on the modal expansion method and Green's function method [8]. Meriç et al. designed a resonator system with a helical side branch duct to control broadband noise [9]. Lu et al. studied a small-scale series-parallel coupling mode broadband microperforated muffler [10]. Yasuda et al. proposed a method for making holes in the tailpipe to improve the muffler performance. The muffler can then simultaneously suppress low-frequency and intermediate-frequency noise [11]. Xiang et al. designed a multicavity microperforated muffler with adjustable transmission loss to reduce the noise of the blower [12]. Zhang et al. used the principle of split-stream rushing to reduce the air velocity of the muffler and analyzed the acoustic performance and flow field in the pipeline. These studies improved the muffler performance in a specific frequency range by improving the overall structure, shape, and size parameters of the prototype muffler [13]. Li et al. proposed a numerical simulation method that comprehensively considered dipole and quadrupole sources to predict aerodynamic noise [14]. Tu et al. based on VOF multiphase flow and Schnerr-Sauer cavitation model, established LES/FW-H coupling model to study the noise characteristics of underwater high-speed vehicle [15]. Yang et al. established a onedimensional computational fluid dynamics model integrating the intake/exhaust system and the engine based on a vehicle with an in-line four-cylinder engine, and predicted the intake noise [16].

With regard to bionic drag reduction and noise reduction, Gruschka et al. conducted an experimental study on the aeroacoustic performance of gliding owls using sensors in a reverberation room [17]. Walsh et al. designed a nonsmooth surface with microscale microprotrusions and used it on an aircraft for experiments [18]. In recent years, Bachmann et al. found that the serrated structure of barn owl wings plays an important role in airflow control and noise reduction [19]. Wang et al. carried out a bionic optimization design of a NACA 0012 airfoil through numerical simulation [20]. Zhang et al. designed a structure on tractor bionic exhaust tailpipes [21]. Zhang et al. designed the inner wall of the tractor exhaust tailpipe by using the bionics method and studied the noise reduction effect of the tractor exhaust tailpipe with different texture shapes [22]. The results showed that the bionic texture has the effect of reducing exhaust liner noise. However, size optimization and experimental verification are not carried out in this paper.

Roger et al. [23], Chong et al. [24], and Wang et al. [25]. carried out numerical simulation research on bionic airfoils with sawtooth structures. Liu et al. studied multiple-coupled bionic blades [26]. Li et al. [27] used the LES method and FW-H equation to numerically simulate the flow field and noise research so that the unsteady pressure fluctuations on the surface of a bionic blade constructed with long-eared owl wing models were suppressed, and the aerodynamic noise was reduced. Liu et al. simulated the influence of aerodynamic noise of vehicle side windows with bionic structures [28]; Dean et al. reviewed the research of shark-skin surfaces for turbulent drag reduction and [29]; Shi et al. reduced the radiated aerodynamic noise of a cylindrical tube structure by mimicking the jagged structure of the leading edge of a long-eared owl wing [30].

To optimize the pipeline noise reduction model, Chang et al. used a neural network and genetic algorithm to optimize the structure of multi-chamber plenum. In the limited space, the maximum noise reduction effect of the hydrostatic chamber is maximized, and the size of the multi-cavity hydrostatic chamber is greatly changed [31]. Jang et al. optimized the structure of a bias inlet muffler through topology optimization design. In order to achieve the purpose of noise reduction, the optimized partition layout of the bias inlet/outlet muffler is systematically designed. The method is verified by experiments [32]. Lee [33] optimized the muffler through the topology optimization method and set a rigid baffle inside the muffler to improve the muffler's performance within the target frequency range and carried out experimental verification. The topology optimization method can achieve good noise reduction in the target frequency range. However, 
the topology structure optimized will have the problem of unclear boundary, so it is not applicable to the structure optimization of the bionic exhaust tailpipe. Chiu et al. obtained the maximum transmission loss of multi-chamber muffler and static pressure chamber at the target frequency by using a genetic algorithm. In order to verify the reliability of genetic algorithm optimization, the optimal noise reduction experiment of one chamber plug inlet muffler was carried out [34,35]. Xu et al. used the boundary element method to optimize the distribution of porous material layer in the cavity to improve the absorption effect of porous material, reduce the noise level or increase the dissipated sound energy of porous material [36]. To sum up, the previously reported literature mainly focuses on the structural optimization design of cavity or multi-cavity materials, but does not study the structural optimization of the exhaust tailpipe of a tractor.

In this paper, a bionic triangular convex texture was applied to the noise reduction of the exhaust tailpipe of a tractor. The noise reduction effect was studied by analyzing its aeroacoustics and transmission loss. Based on a BP neural network optimized by a genetic algorithm, the structural parameters of the bionic triangular convex textures were optimized to obtain the maximum transmission loss. Then, the noise reduction performance was experimentally verified.

\section{Materials and Methods}

\subsection{Establishment and Meshing of Bionic Model}

The nonsmooth surface of a shark has an effect on reducing resistance [37]. As shown in Figure 1a [38], the surface of a shark with triangular grooves has the function of drag reduction, which provides a basis for research on drag reduction and noise reduction. Inspired by the surface of shark skin, Walsh et al. designed a sawtooth riblet structure, as shown in Figure 1b [29], and performed many experimental studies [39,40]. The research showed that the isosceles triangular groove has the best drag reduction performance. In this paper, the bionic exhaust tailpipe of a tractor was designed, as shown in Figure 1c. Bionic triangular convex textures were added to the inner wall surface of the tractor exhaust tailpipe. The top angle $\theta$ of the triangular texture was selected from $30^{\circ}, 45^{\circ}, 60^{\circ}, 75^{\circ}$, and $90^{\circ}$, in sequence. The texture height $\mathrm{h}$ was selected from $0.5,0.75,1,1.25$, and $1.5 \mathrm{~mm}$. The number of circumferential columns $\mathrm{n}$ of the texture was selected from 18, 24, 30, and 36. The length of the triangular texture along the inner wall of the tube was $384 \mathrm{~mm}$. A three-dimensional diagram of the air cavity of the prototype and bionic exhaust tailpipe is shown in Figure 2.

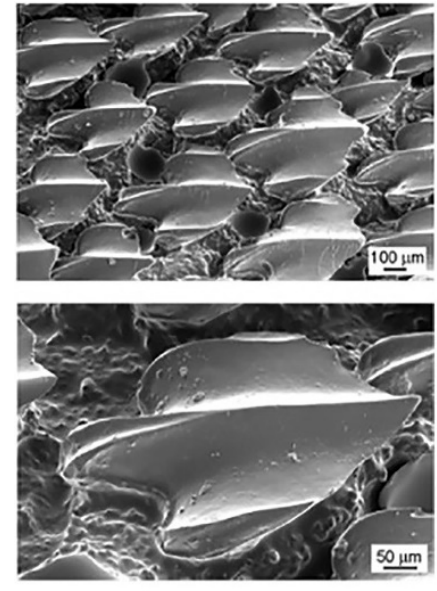

(a)

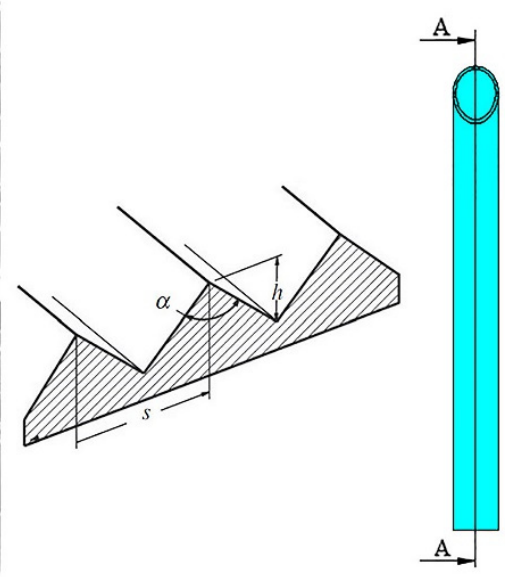

(b)
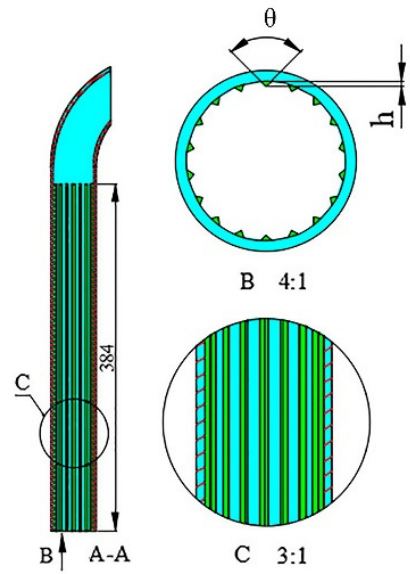

B $4: 1$

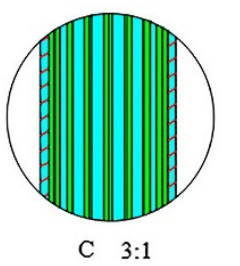

(c)

Figure 1. Bionic model diagram: (a) SEM micrographs of shark-skin (Squalus acanthias) replica [38], Reproduced with the permission of ref. [38], copyright@ J. Phys. Condens. Matter, 2010. (b) schematic of sawtooth riblets bioinspired by shark-skin surfaces [29], Reproduced with the permission of ref. [29], copyright@ Philos. T. R. Soc. A, 2010. (c) tractor bionic exhaust tailpipe. 


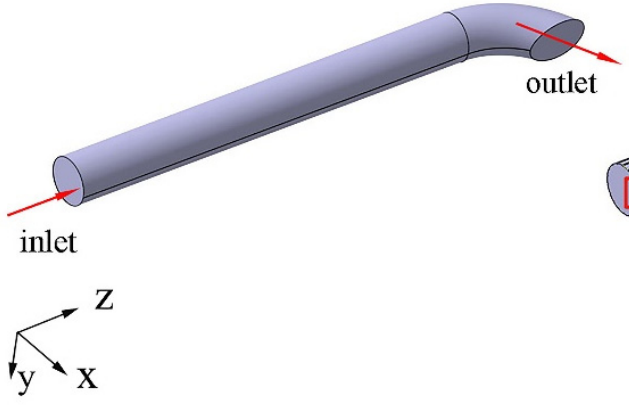

(a)

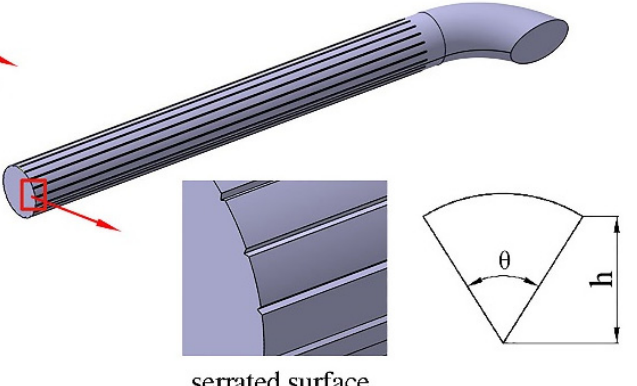

(b)

Figure 2. Three-dimensional diagram of air cavity: (a) prototype and (b) bionic exhaust tailpipe.

The grid division of computational aeroacoustics is shown in Figure 3a,b. To make accurate calculations, a hexahedral structural grid was used for mesh generation. The number of total grids of the prototype tailpipe was approximately $1,831,074$, and that of the bionic tailpipe was approximately 7,000,000. To calculate the transmission loss of the exhaust tailpipe, the sound field was analyzed with LMS Virtual Lab software. The condition that the side length of the grid unit should satisfy is $L \leq c /\left(6 f_{\max }\right)$ [41]. $c$ is the propagation speed of sound in the fluid medium, $f_{\max }$ is the highest calculation frequency, and $L$ is the length of the unit to be calculated. The air cavity grids of the prototype and bionic exhaust tailpipe are shown in Figure $3 c, d$, where the maximum unit size is $10 \mathrm{~mm}$ and the minimum unit size is $0.5 \mathrm{~mm}$.

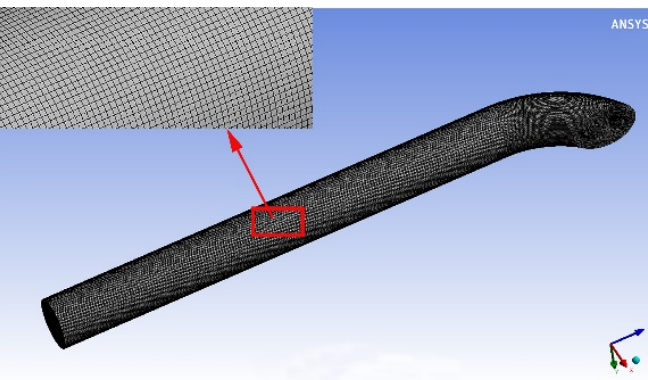

(a)

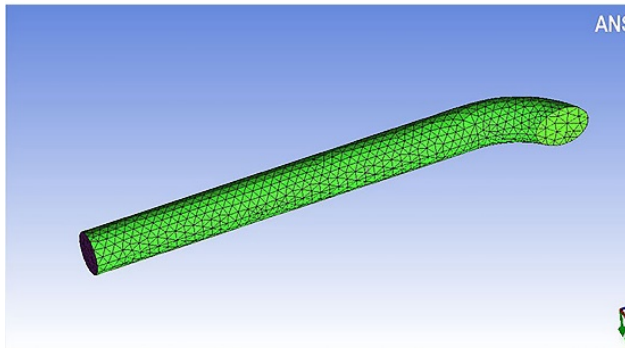

(c)

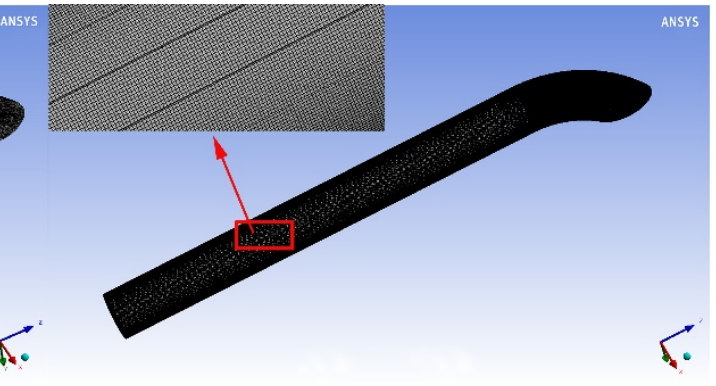

(b)

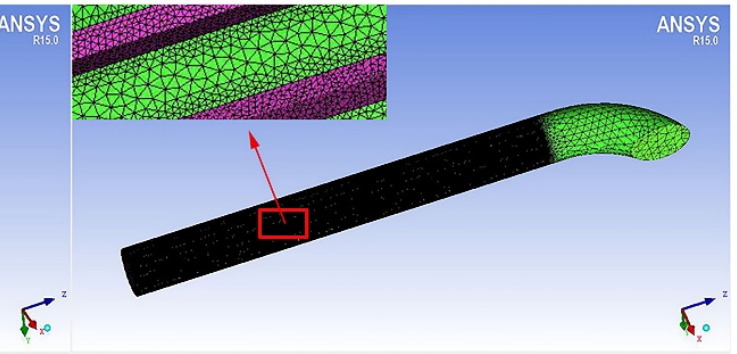

(d)

Figure 3. Air cavity grid of exhaust tailpipe: (a) prototype, (b) bionic exhaust pipe used for aeroacoustics, (c) prototype used for transmission loss, (d) bionic exhaust pipe used for transmission loss.

\subsection{Boundary Conditions and Acoustic Solution Settings}

In this research, the simulation software ANSYS Fluent was used to calculate the internal flow field of the exhaust tailpipe to predict the aerodynamic noise generated in the exhaust tailpipe and analyze the noise reduction mechanism of the bionic exhaust tailpipe. First, a large eddy simulation (LES) was used to solve the Navier-Stokes equations, and then the sound pressure level at the outlet monitoring point was calculated by solving the Ffowcs Williams and Hawkings (FW-H) equation. The sound pressure level (SPL) spectrum at the monitoring point was obtained by fast Fourier transform (FFT), and the 
reference pressure was $2 \times 10^{-5} \mathrm{~Pa}$. The time step size was set to $0.0002 \mathrm{~s}$, and the upper limit frequency was $2500 \mathrm{~Hz}$ to calculate the aeroacoustics. The simulation software LMS Virtual Lab Acoustics FEM was used to perform simulation analysis. The fluid was air, its density was $1.225 \mathrm{~kg} / \mathrm{m}^{3}$, and the velocity of the sound in the fluid was $340 \mathrm{~m} / \mathrm{s}$. The inlet boundary conditions were set as follows. The vibration velocity was $1 \mathrm{~m} / \mathrm{s}$, and the fluid velocity was $50 \mathrm{~m} / \mathrm{s}$. The full sound absorption property was set at the outlet to simulate no reflection boundary condition. The acoustic impedance of the fluid was $\rho_{0} c=416.5 \mathrm{~kg} /\left(\mathrm{m}^{2} \cdot \mathrm{s}\right)$, the calculation frequency was $10 \mathrm{~Hz}$ to $1000 \mathrm{~Hz}$, and the calculation interval step was $20 \mathrm{~Hz}$.

The governing equations for LES are obtained by filtering Navier-Stokes equations in wavenumber space or physical space. The filter variable is defined as:

$$
\bar{\varphi}(x)=\int_{D} \varphi\left(x^{\prime}\right) G\left(x, x^{\prime}\right) d x^{\prime}
$$

where $D$ is the flow area, $\varphi$ is a transient variable, $x^{\prime}$ is the actual flow area with spatial coordinates, and $x$ is the spatial coordinates in the filtered large-scale space. $G$ is the filter equation. The filter function to average physical quantities on the control volume is defined as:

$$
\bar{\varphi}(x)=\frac{1}{V} \int_{V} \varphi\left(x^{\prime}\right) d x^{\prime}, x^{\prime} \in v
$$

where $V$ is the volume of the calculated unit.

$$
G\left(x, x^{\prime}\right)=\left\{\begin{array}{l}
1 / V, x^{\prime} \in v \\
0, x^{\prime} \notin v
\end{array}\right.
$$

The transient Navier-Stokes equation and continuous equation are processed by the filter function.

$$
\begin{gathered}
\frac{\partial(\rho)}{\partial t}+\frac{\partial\left(\rho \bar{u}_{i}\right)}{\partial x_{i}}=0 \\
\frac{\partial\left(\rho \bar{u}_{i}\right)}{\partial t}+\frac{\partial\left(\rho \bar{u}_{i} \bar{u}_{j}\right)}{\partial x_{j}}=\frac{\partial\left(\sigma_{i j}\right)}{\partial x_{j}}-\frac{\partial \bar{p}}{\partial x_{i}}-\frac{\partial \tau_{i j}}{\partial x_{j}}
\end{gathered}
$$

where $\rho$ is the air density, $\bar{u}_{i}$ is the filter velocity component $x_{i}$ in the Cartesian coordinate system, $\bar{p}$ is the filter pressure, $\sigma_{i j}$ is the stress tensor caused by molecular viscosity, and $\tau_{i j}$ is the subgrid scale stress.

$$
\begin{gathered}
\sigma_{i j} \equiv\left[\mu\left(\frac{\partial \bar{u}_{i}}{\partial x_{j}}+\frac{\partial \bar{u}_{j}}{\partial x_{i}}\right)\right]-\frac{2}{3} \mu \frac{\partial \bar{u}_{i}}{\partial x_{j}} \delta_{i j} \\
\tau_{i j}=\rho \bar{u}_{i} \bar{u}_{j}-\rho \bar{u}_{i} \bar{u}_{j}
\end{gathered}
$$

In this research, the turbulent eddy viscosity is defined by the Wall-Adapting Local Eddy-Viscosity Model (WALE). In the WALE model, the SGS stress can be expressed as:

$$
\tau_{i j}-\frac{1}{3} \tau_{k k} \delta_{i j}=-2 \mu_{t} \bar{S}_{i j}
$$

The eddy viscosity is modeled as:

$$
\mu_{t}=\rho \Delta_{s}{ }^{2} \frac{\left(S_{i j}{ }^{d} S_{i j}{ }^{d}\right)^{3 / 2}}{\left(\bar{S}_{i j} \bar{S}_{i j}\right)^{5 / 2}+\left(S_{i j}{ }^{d} S_{i j}{ }^{d}\right)^{5 / 4}}
$$

where $\Delta_{s}$ is the filter size, and $\Delta_{s}=C_{w} V^{1 / 3}$. 
The model parameter $C_{w}$ is set to $0.325, S_{i j}^{d}=\frac{1}{2}\left(\bar{g}_{i j}^{2}+\bar{g}_{j i}^{2}\right)-\frac{1}{3} \delta_{i j} \bar{g}_{k k}^{2}, \bar{g}_{i j}=\frac{\partial \bar{u}_{i}}{\partial x_{j}}$, and $\overline{S_{i j}}$ is the rate of the strain tensor for the resolved scale defined by:

$$
\bar{S}_{i j}=\frac{1}{2}\left(\frac{\partial \bar{u}_{i}}{\partial x_{j}}+\frac{\partial \bar{u}_{j}}{\partial x_{i}}\right)
$$

The FW-H acoustic equation can be expressed as [42]:

$$
\frac{1}{a_{0}^{2}} \frac{\partial^{2} p^{\prime}}{\partial t^{2}}-\nabla^{2} p^{\prime}=\frac{\partial^{2}}{\partial x_{i} \partial x_{j}}\left\{T_{i j} H(f)\right\}-\frac{\partial}{\partial x_{i}}\left\{\left[p_{i j} n_{j}+\rho u_{i}\left(u_{n}-v_{n}\right)\right] \delta(f)\right\}+\frac{\partial}{\partial t}\left\{\left[p_{0} v_{n}+\rho\left(u_{n}-v_{n}\right)\right] \delta(f)\right\}
$$

where $\delta(f)$ is the Dirac delta function, and $H(f)$ is the Heaviside function. In this paper, $p^{\prime}$ is the far-field sound pressure $\left(p^{\prime} \equiv p-p_{0}\right), T_{i j}$ is the Lighthill stress tensor, and $p_{i j}$ is the compressible stress tensor.

The total sound pressure level calculation equation is defined as

$$
L_{p t}(d B)=10 \log _{10} \sum_{i=1}^{n} 10^{\left(\frac{L_{p i}}{10}\right)}
$$

where $L_{p i}$ is the sound pressure level at the corresponding frequency, and $i$ is the number of frequencies. The flow velocity in this paper is less than 0.3 Mach number. In this case, the calculation of aerodynamic noise only considers the dipole noise caused by fluctuating pressure on the model surface.

The noise of the tailpipe includes aerodynamic noise and vibration noise. The vibration noise generated by internal combustion engines was simulated by applying unit particle vibration velocity at the inlet of the exhaust tailpipe, and the noise reduction effect of the bionic exhaust tailpipe was studied.

The transmission loss is the difference between the incident sound power level at the inlet of the muffler and the radiated sound power level at the outlet when there is no reflection end at the outlet. The transmission loss was calculated by Equation (13):

$$
T L(d B)=L_{W_{\text {in }}}-L_{W_{\text {out }}}=10 \lg \left(\frac{W_{\text {in }}}{W_{\text {out }}}\right)=10 \lg \left(\frac{p_{1} \overline{p_{1}}}{p_{2} \overline{\overline{p_{2}}}} \frac{A_{\text {in }}}{A_{\text {out }}}\right)
$$

where $L_{W_{\text {in }}}$ is the incident sound power level, $L_{W_{\text {out }}}$ is the radiate sound power level, $A_{\text {in }}$ is the inlet cross-sectional area, and $A_{\text {out }}$ is the outlet cross-sectional area.

$$
\begin{gathered}
p_{1}=\frac{1}{2}\left(p_{\text {inlet }}+\rho_{0} c\right) \\
p_{2}=p_{\text {outlet }}
\end{gathered}
$$

where $P_{\text {inlet }}$ is the sound pressure at the inlet, $P_{\text {outlet }}$ is the sound pressure at the outlet, $\rho_{0} c$ is the acoustic impedance, $p_{1}$ and $\overline{p_{1}}$ are conjugate complex numbers, and $p_{2}$ and $\overline{p_{2}}$ are conjugate complex numbers.

The formula of transmission loss is:

$$
T L(d B)=10 \lg \left(\frac{A_{\text {in }}}{A_{\text {out }}} \times \frac{p_{1} \overline{p_{1}}}{p_{2} \overline{p_{2}}}\right)
$$

The ratios $A=A_{\text {in }} / A_{\text {out }}$ are shown in Table 1 and show the dimension parameters of the bionic exhaust tailpipes during the simulation calculation. 
Table 1. Cross-sectional area ratios of inlet and outlet under different size parameters ( $\theta$ is top angle, $\mathrm{h}$ is texture height, $\mathrm{n}$ is the number of circumferential columns, and $A$ is $A_{\text {in }} / A_{\text {out }}$ ratio).

\begin{tabular}{|c|c|c|c|c|c|c|c|c|c|c|c|}
\hline \multicolumn{3}{|c|}{$\mathrm{n}=\mathbf{1 8}$} & \multicolumn{3}{|c|}{$\mathrm{n}=24$} & \multicolumn{3}{|c|}{$\mathrm{n}=30$} & \multicolumn{3}{|c|}{$\mathrm{n}=36$} \\
\hline$\theta\left({ }^{\circ}\right)$ & $\mathrm{h}(\mathrm{mm})$ & $A$ & $\theta\left(^{\circ}\right)$ & $\mathrm{h}(\mathrm{mm})$ & $A$ & $\theta\left(^{\circ}\right)$ & $\mathrm{h}(\mathrm{mm})$ & $A$ & $\theta\left({ }^{\circ}\right)$ & $\mathrm{h}(\mathrm{mm})$ & $A$ \\
\hline 30 & 0.5 & 0.80 & 30 & 0.5 & 0.80 & 30 & 0.5 & 0.80 & 30 & 0.5 & 0.80 \\
\hline 30 & 0.75 & 0.80 & 30 & 0.75 & 0.80 & 30 & 0.75 & 0.80 & 30 & 0.75 & 0.80 \\
\hline 30 & 1 & 0.80 & 30 & 1 & 0.79 & 30 & 1 & 0.79 & 30 & 1 & 0.79 \\
\hline 30 & 1.25 & 0.79 & 30 & 1.25 & 0.79 & 30 & 1.25 & 0.79 & 30 & 1.25 & 0.79 \\
\hline 30 & 1.5 & 0.79 & 30 & 1.5 & 0.79 & 30 & 1.5 & 0.79 & 30 & 1.5 & 0.79 \\
\hline 45 & 0.5 & 0.80 & 45 & 0.5 & 0.80 & 45 & 0.5 & 0.80 & 45 & 0.5 & 0.80 \\
\hline 45 & 0.75 & 0.80 & 45 & 0.75 & 0.79 & 45 & 0.75 & 0.79 & 45 & 0.75 & 0.79 \\
\hline 45 & 1 & 0.79 & 45 & 1 & 0.79 & 45 & 1 & 0.79 & 45 & 1 & 0.79 \\
\hline 45 & 1.25 & 0.79 & 45 & 1.25 & 0.79 & 45 & 1.25 & 0.79 & 45 & 1.25 & 0.79 \\
\hline 45 & 1.5 & 0.79 & 45 & 1.5 & 0.79 & 45 & 1.5 & 0.78 & 45 & 1.5 & 0.78 \\
\hline 60 & 0.5 & 0.80 & 60 & 0.5 & 0.80 & 60 & 0.5 & 0.80 & 60 & 0.5 & 0.80 \\
\hline 60 & 0.75 & 0.79 & 60 & 0.75 & 0.79 & 60 & 0.75 & 0.79 & 60 & 0.75 & 0.79 \\
\hline 60 & 1 & 0.79 & 60 & 1 & 0.79 & 60 & 1 & 0.79 & 60 & 1 & 0.79 \\
\hline 60 & 1.25 & 0.79 & 60 & 1.25 & 0.79 & 60 & 1.25 & 0.78 & 60 & 1.25 & 0.78 \\
\hline 60 & 1.5 & 0.79 & 60 & 1.5 & 0.78 & 60 & 1.5 & 0.78 & 60 & 1.5 & 0.77 \\
\hline 75 & 0.5 & 0.80 & 75 & 0.5 & 0.80 & 75 & 0.5 & 0.79 & 75 & 0.5 & 0.79 \\
\hline 75 & 0.75 & 0.79 & 75 & 0.75 & 0.79 & 75 & 0.75 & 0.79 & 75 & 0.75 & 0.79 \\
\hline 75 & 1 & 0.79 & 75 & 1 & 0.79 & 75 & 1 & 0.79 & 75 & 1 & 0.78 \\
\hline 75 & 1.25 & 0.79 & 75 & 1.25 & 0.78 & 75 & 1.25 & 0.78 & 75 & 1.25 & 0.78 \\
\hline 75 & 1.5 & 0.78 & 75 & 1.5 & 0.78 & 75 & 1.5 & 0.77 & 75 & 1.5 & 0.77 \\
\hline 90 & 0.5 & 0.80 & 90 & 0.5 & 0.79 & 90 & 0.5 & 0.79 & 90 & 0.5 & 0.79 \\
\hline 90 & 0.75 & 0.79 & 90 & 0.75 & 0.79 & 90 & 0.75 & 0.79 & 90 & 0.75 & 0.79 \\
\hline 90 & 1 & 0.79 & 90 & 1 & 0.79 & 90 & 1 & 0.78 & 90 & 1 & 0.78 \\
\hline 90 & 1.25 & 0.78 & 90 & 1.25 & 0.78 & 90 & 1.25 & 0.77 & 90 & 1.25 & 0.77 \\
\hline 90 & 1.5 & 0.78 & 90 & 1.5 & 0.77 & 90 & 1.5 & 0.76 & 90 & 1.5 & 0.76 \\
\hline
\end{tabular}

\subsection{Parameter Optimization Algorithm}

The artificial neural network model (ANN) [43], which is also known as a neural network (NN), is a mathematical model of an algorithm that imitates the behavioral characteristics of animal neural networks for distributed parallel information processing. As a kind of artificial neural network, a BP neural network is a multilayer feeding forward neural network that includes an input layer, hidden layer, and output layer. The BP neural network algorithm consists of two processes: forward propagation of the signal and backward propagation of the error signal.

BP neural network signals forward propagation process as follows:

Equation (17) represents the input of the $i$-th node of the hidden layer [44]:

$$
\text { net }_{i}=\sum_{j=1}^{M} \omega_{i j} x_{j}+\theta_{i}
$$

where $x_{j}$ is the input of the $j$-th node of the input layer, and $j$ ranges from 1 to $M$. $\omega_{i j}$ is the weight from the $i$-th node of the hidden layer to the $j$-th node of the input layer, and $\theta_{i}$ is the threshold of the $i$-th node of the hidden layer.

Equation (18) represents the output of the $i$-th node of the hidden layer:

$$
\mathrm{o}_{i}=\phi\left(\text { net }_{i}\right)=\phi\left(\sum_{j=1}^{M} \omega_{i j} x_{j}+\theta_{i}\right)
$$

where $\phi\left(\right.$ net $\left._{i}\right)$ is the excitation function of the hidden layer. 
Equation (19) represents the output of the $k$-th node of the output layer:

$$
\text { net }_{i}=\sum_{j=1}^{M} \omega_{i j} x_{j}+\theta_{i} \text { net }_{k}=\sum_{i=1}^{q} \omega_{k i} y_{i}+a_{k}=\sum_{i=1}^{q} \omega_{k i} \phi\left(\sum_{j=1}^{M} \omega_{i j} x_{i}+\theta_{i}\right)+a_{k}
$$

where $\omega_{k i}$ is the weight from the $k$-th node of the input layer to the $i$-th node of hidden layer $i$ ranging from 1 to $q . a_{k}$ is the threshold of the $k$-th node of the input layer, and $k$ ranges from 1 to $L$.

Equation (20) represents the output of the $k$-th node of the output layer:

$$
o_{k}=\psi\left(\text { net }_{k}\right)=\psi\left[\sum_{i=1}^{q} \omega_{k i} \phi\left(\sum_{j=1}^{M} \omega_{i j} x_{j}+\theta_{i}\right)+a_{k}\right]
$$

where $\psi\left(\right.$ net $\left._{k}\right)$ is the excitation function of the input layer, and $o_{k}$ is the output of the $k$-th node of the output layer.

Back propagation of errors as follows:

Equation (21) indicates that the quadratic error criterion function for each sample is:

$$
E_{P}=\frac{1}{2} \sum_{k=1}^{L}\left(T_{k}-o_{k}\right)^{2}
$$

where $T_{k}$ is the expected output value.

Equation (22) indicates the total error criterion function of the system training samples:

$$
E_{P}=\frac{1}{2} \sum_{p=1}^{P} \sum_{k=1}^{L}\left(T_{k}^{p}-o_{k}^{p}\right)^{2}
$$

\subsection{Method Descriptions}

\subsubsection{Sample}

To verify the simulation effect in this work, prototype and bionic exhaust tailpipes were manufactured. The bionic tailpipe samples were precisely manufactured by wire cutting. The top angle $\theta$ of the triangular texture was selected from $45^{\circ}, 60^{\circ}$, and $75^{\circ}$ in sequence. The texture height $\mathrm{h}$ was selected from $0.75,1$, and $1.25 \mathrm{~mm}$. The number of circumferential columns $\mathrm{n}$ of the texture was selected as 24 . To facilitate processing, the tail elbow was ignored. The total length of the triangular texture tailpipes was $402 \mathrm{~mm}$, and the triangular texture along the inner wall of the tube was $384 \mathrm{~mm}$. The thickness of the experimental specimen is $3 \mathrm{~mm}$. A three-dimensional diagram of the tailpipe specimens is shown in Figure 4.

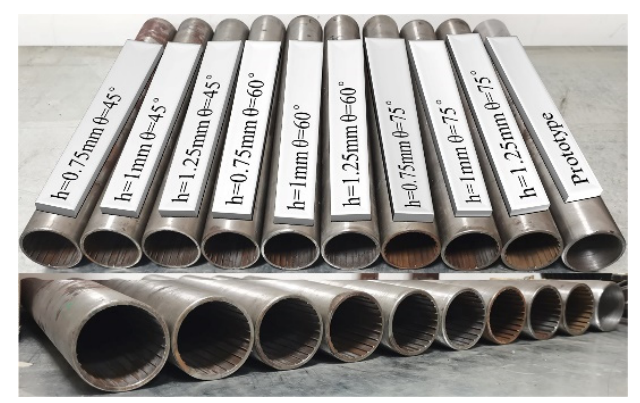

Figure 4. Experimental test specimens.

\subsubsection{Experimental Setup for Insertion Loss}

The simulation analysis could calculate the possibility of bionic exhaust tailpipes with regard to noise reduction. However, it is still unknown how practical implementa- 
tion performs and whether noise will be reduced by bionic exhaust tailpipes. Therefore, experimental tests are carried out to conduct a further evaluation of the comprehensive performance of prototype and bionic exhaust tailpipes, as shown in Figure 5.

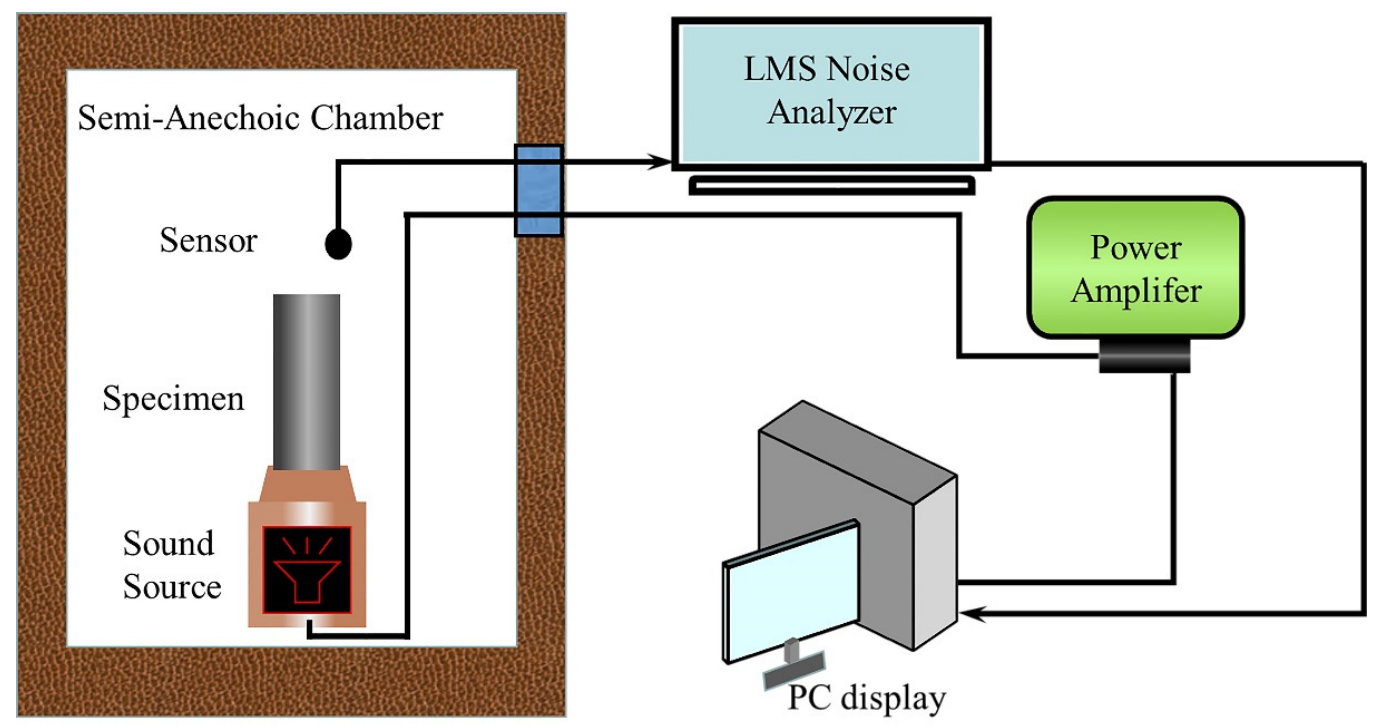

Figure 5. Schematic diagram of the experimental setup of insertion loss.

Figure 5 shows a schematic diagram of the insertion loss test device. The test system consists of a noise generation system, acoustic parameter measurement system, and exhaust tailpipe sample. Using sound sources and power amplifiers can provide noise signals under different frequency conditions as noise sources. In addition, the acoustic measurement system includes microphones (1/2" PCB sensor), LMS vibration and noise analyzer, and acoustic analysis software (LMS Test Lab). In this paper, the test frequency range is set as $15-2500 \mathrm{~Hz}$ during the test, and the sound pressure level curve of the $1 / 3$ octave range at the test point is obtained.

In addition, the insertion loss in this work is caused by the difference between the measured sound pressure levels at the outlet under the conditions of the prototype and bionic tailpipes. Moreover, the insertion loss test is carried out in a semi-anechoic chamber, and the sound source and sensor are placed indoors to reduce the impact of sound reflection and radiation on the experimental tests. Figure 6 shows the experimental setup for insertion loss.

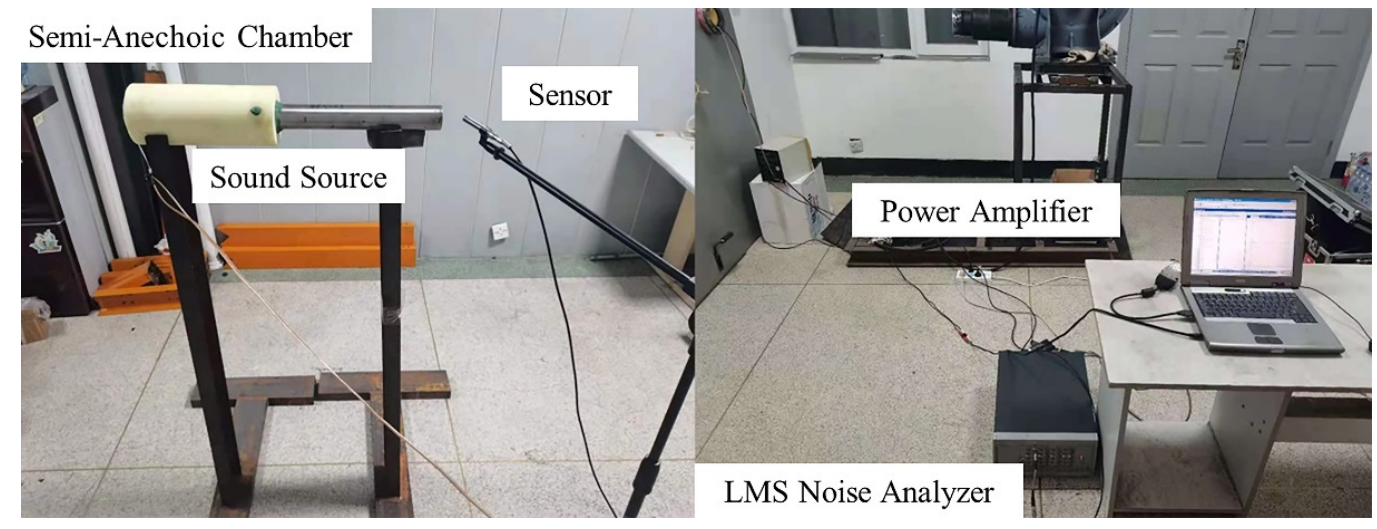

Figure 6. Photograph of experimental setup for insertion loss. 


\section{Results and Discussion}

\subsection{Aeroacoustics and Transmission Loss Analysis}

The sound pressure level frequency response curves of the prototype and bionic exhaust tailpipes at different inlet velocities are shown in Figure 7. The size parameters of the bionic exhaust tailpipe were selected from Table 1 . The top angle $\theta$ was $60^{\circ}$, the texture height $\mathrm{h}$ was $1 \mathrm{~mm}$, and the number of circumferential columns $\mathrm{n}$ was 24 . The inlet airflow velocities of the tailpipe were 40,50,60, and $70 \mathrm{~m} / \mathrm{s}$, and the corresponding Reynolds numbers were $1.20 \times 10^{5}, 1.51 \times 10^{5}, 1.81 \times 10^{5}$, and $2.21 \times 10^{5}$. The results suggested that the sound pressure level gradually increased at the exit monitoring point with increasing air velocity. As shown in Table 2, the total sound pressure level of the prototype tailpipe increased from $111.256 \mathrm{~dB}$ to $119.461 \mathrm{~dB}$, increasing by $8.205 \mathrm{~dB}$. The total sound pressure level of the bionic tailpipe increased from $109.406 \mathrm{~dB}$ to $117.303 \mathrm{~dB}$, increasing by $7.897 \mathrm{~dB}$. The total sound pressure level of the bionic exhaust tailpipe decreased by $1.302 \mathrm{~dB}$ to $2.560 \mathrm{~dB}$ compared with that of the prototype tailpipe. The noise reduction effect was most obvious when the air velocity was $50 \mathrm{~m} / \mathrm{s}$ and the sound pressure level was reduced by $2.560 \mathrm{~dB}$. Yu Liu et al. studied the noise reduction of a wavy multi-copter rotor; the attenuation of total sound pressure level of the wavy rotor with respect to the baseline rotor was about 1.4-2 dB [45]. Aerodynamic and acoustic investigations of multi-copter rotors with trailing edge serrations have been performed. The results suggested that the serrated rotor had a total sound pressure level attenuation of $0.9-1.6 \mathrm{~dB}[46,47]$. The noise reduction effect through leading-edge serrations was studied on two-dimensional airfoils, and alleviated the total sound pressure level of $1.5 \mathrm{~dB}$ [48]. Therefore, the role played by the bionic exhaust tailpipe in this paper achieved a significant effect in aeroacoustics. At different inlet airflow velocities, the total sound pressure level of the bionic tailpipe was decreased compared with that of the prototype tailpipe. The results showed that the bionic tailpipe reduced the aerodynamic noise. The main reason was that the bionic texture could suppress the turbulent flow near the wall of the tailpipe and reduce aerodynamic drag and aerodynamic noise. The noise reduction performance of $70 \mathrm{~m} / \mathrm{s}$ is worse than $60 \mathrm{~m} / \mathrm{s}$, mainly due to the increase of airflow velocity, which leads to the increase of airflow regeneration noise. The sound wave at high frequency propagates in the form of non-plane wave, which leads to more obvious airflow regeneration noise. Therefore, the performance of the bionic exhaust tailpipe is better at $60 \mathrm{~m} / \mathrm{s}$ than $70 \mathrm{~m} / \mathrm{s}$, especially in the 2000-2500 Hz range.

To research the influence of size parameters on the aerodynamic noise reduction performance of bionic tailpipes, different size parameters of bionic tailpipes were simulated, as shown in Figures 8 and 9. As shown in Figures 8a and 9a, the noise reduction effect gradually increased, and the total sound pressure level of bionic tailpipes gradually reduced as the circumferential column number $\mathrm{n}$ increased from 18 to 36 . The total sound pressure level of bionic tailpipes was reduced by $1.901 \mathrm{~dB}-2.790 \mathrm{~dB}$ compared with the prototype tailpipe. There was more low-speed airflow near the wall of the bionic tailpipe with an increase in the number of circumferential columns $n$, which produced a better drag reduction effect and reduced the generation of aerodynamic noise. In addition, as shown in Figures $8 \mathrm{~b}$ and $9 \mathrm{~b}$, the total sound pressure level of the bionic tailpipes decreased by $0.223 \mathrm{~dB}-1.911 \mathrm{~dB}$ compared with the prototype tailpipe with increasing texture height $\mathrm{h}$. The total sound pressure level of bionic tailpipes first decreased and then increased. This indicates that the form of secondary vortices near the texture can suppress the generation of turbulence, which was the main reason for reducing aerodynamic noise. As the height reached a certain level, the airflow drag inside the tailpipe increased correspondingly with increasing texture height, which weakened the noise reduction effect. As shown in Figures $8 \mathrm{c}$ and $9 \mathrm{c}$, the influence of the top angle $\theta$ had little impact on the sound pressure level of bionic tailpipes, which indicated that the top angle $\theta$ was not the main factor in reducing aerodynamic noise. 

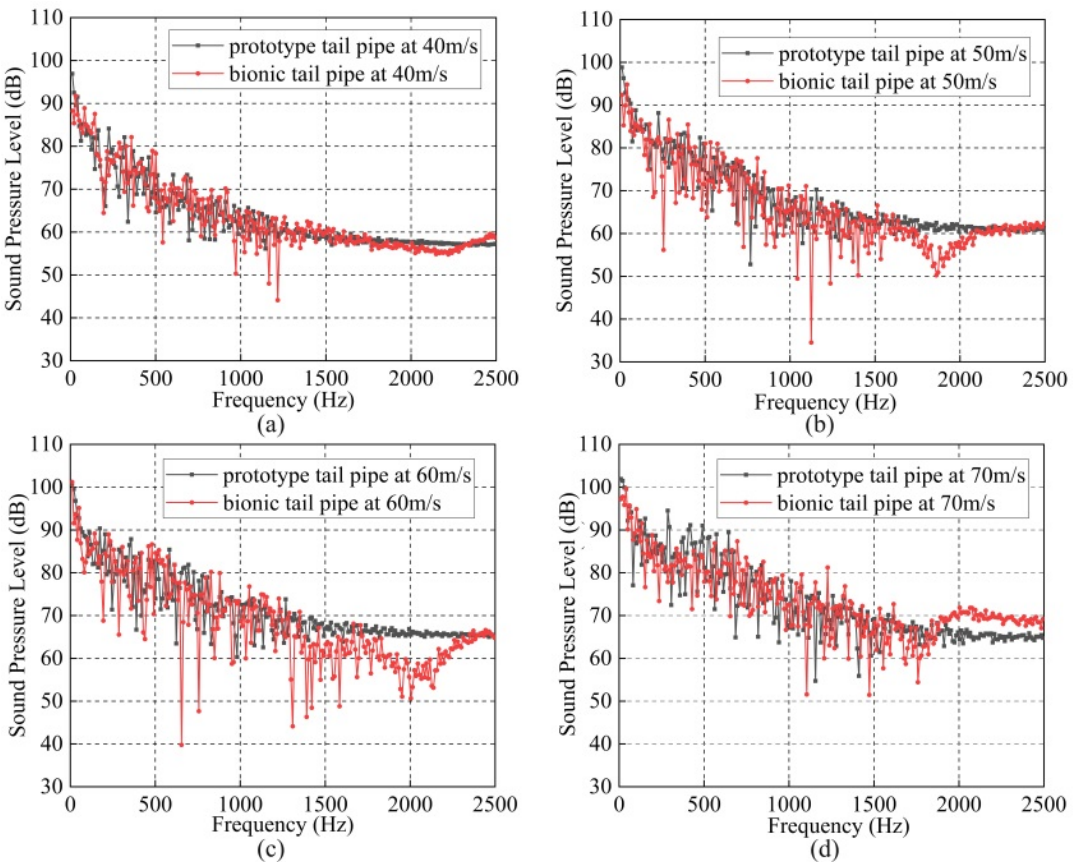

Figure 7. Sound pressure level frequency response curve of prototype and bionic exhaust tailpipe at different airflow velocities: (a) at $40 \mathrm{~m} / \mathrm{s},(\mathbf{b})$ at $50 \mathrm{~m} / \mathrm{s},(\mathbf{c})$ at $60 \mathrm{~m} / \mathrm{s},(\mathbf{d})$ at $70 \mathrm{~m} / \mathrm{s}$.

Table 2. Total sound pressure level between $10 \mathrm{~Hz}$ and $2500 \mathrm{~Hz}$.

\begin{tabular}{cccc}
\hline \multirow{2}{*}{ Velocity $(\mathrm{m} / \mathbf{s})$} & \multicolumn{3}{c}{ Total Sound Pressure Level (dB) } \\
\cline { 2 - 4 } & Prototype & Bionic Tailpipes & Difference \\
\hline 40 & 111.256 & 109.406 & 1.850 \\
50 & 114.233 & 111.673 & 2.560 \\
60 & 116.724 & 115.422 & 1.302 \\
70 & 119.461 & 117.303 & 2.158 \\
\hline
\end{tabular}

The vibration noise reduction performance of the bionic exhaust tailpipes was studied by calculating the transmission loss. The transmission loss curves of the bionic exhaust tailpipes with different top angles, heights, and numbers of circumferential columns were analyzed to study the regular changes in the size parameters of the bionic triangle texture and the transmission loss. The transmission loss of the bionic exhaust tailpipe was compared with that of the prototype exhaust tailpipe.

A comparative analysis of transmission loss curves between the bionic exhaust tailpipes with different triangular texture parameters and the prototype exhaust tailpipe is shown in Figure 10. As seen from the transmission loss curve of the prototype exhaust tailpipe, between $10 \mathrm{~Hz}$ and $50 \mathrm{~Hz}$, the transmission loss is relatively high: between $5.288 \mathrm{~dB}$ and $19.745 \mathrm{~dB}$. From $70 \mathrm{~Hz}$ to $110 \mathrm{~Hz}$, the transmission loss is between $0.752 \mathrm{~dB}$ and $2.897 \mathrm{~dB}$. The noise reduction effect decreases significantly when the frequency is between $130 \mathrm{~Hz}$ and $410 \mathrm{~Hz}$, and the transmission loss is between $0.035 \mathrm{~dB}$ and $0.418 \mathrm{~dB}$. Above $430 \mathrm{~Hz}$, the transmission loss becomes negative, and the noise reduction effect is the worst. As a whole, the transmission loss of the prototype exhaust tailpipe decreases with increasing frequency. Since diesel engine exhaust noise is mainly low frequency and medium frequency [49], the target frequency of this paper is between $10 \mathrm{~Hz}$ and $490 \mathrm{~Hz}$. The transmission loss of the prototype exhaust tailpipe within the target frequency range is relatively low, and the noise reduction effect is not very good and needs to be improved. 


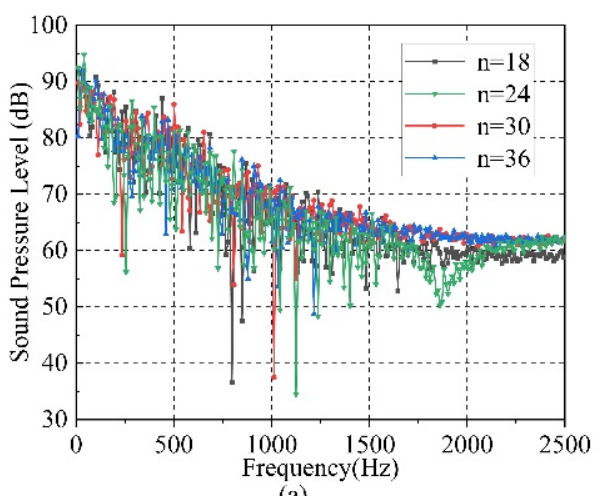

(a)

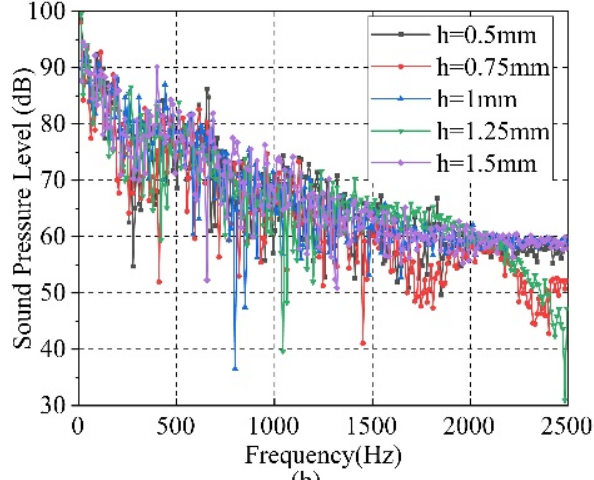

(b)

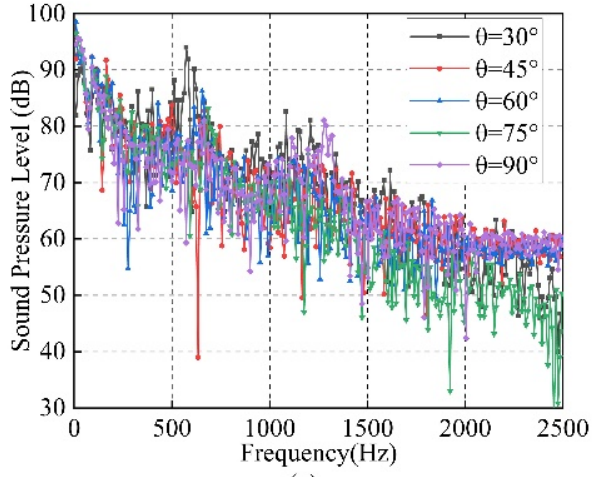

(c)

Figure 8. Effect of dimension parameters on sound pressure level of bionic tailpipes: (a) circumferential column number $\mathrm{n},(\mathbf{b})$ texture height $\mathrm{h},(\mathbf{c})$ top angle $\theta$.

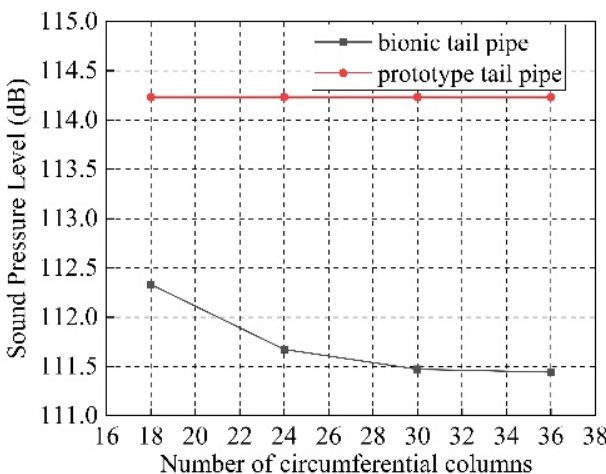

(a)

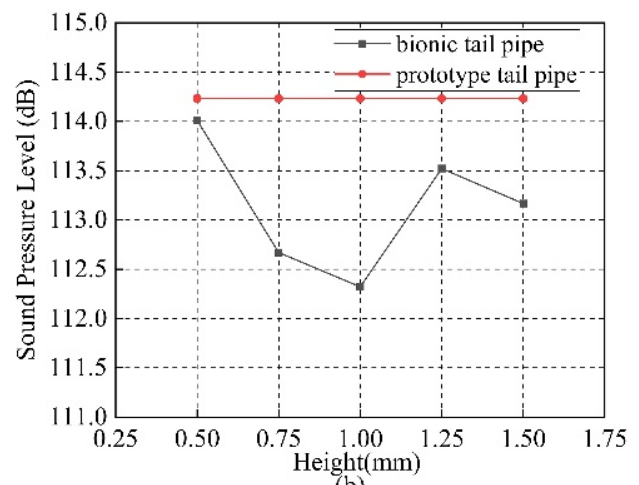

(b)

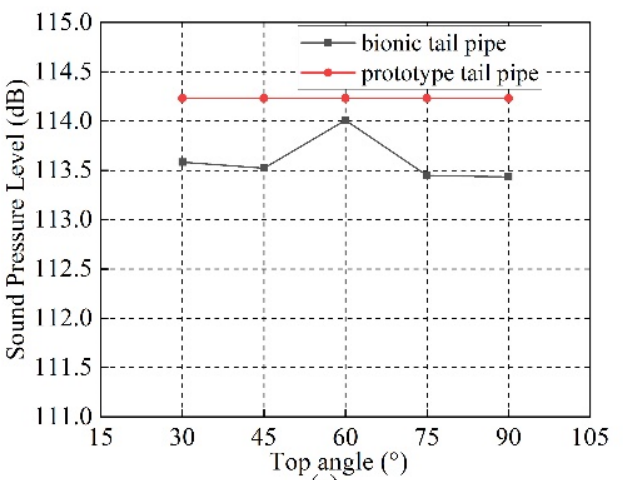

(c)

Figure 9. Effect of dimension parameters on total sound pressure level of bionic tailpipes: (a) circumferential column number $\mathrm{n}$, (b) texture height $\mathrm{h}$, (c) top angle $\theta$. 

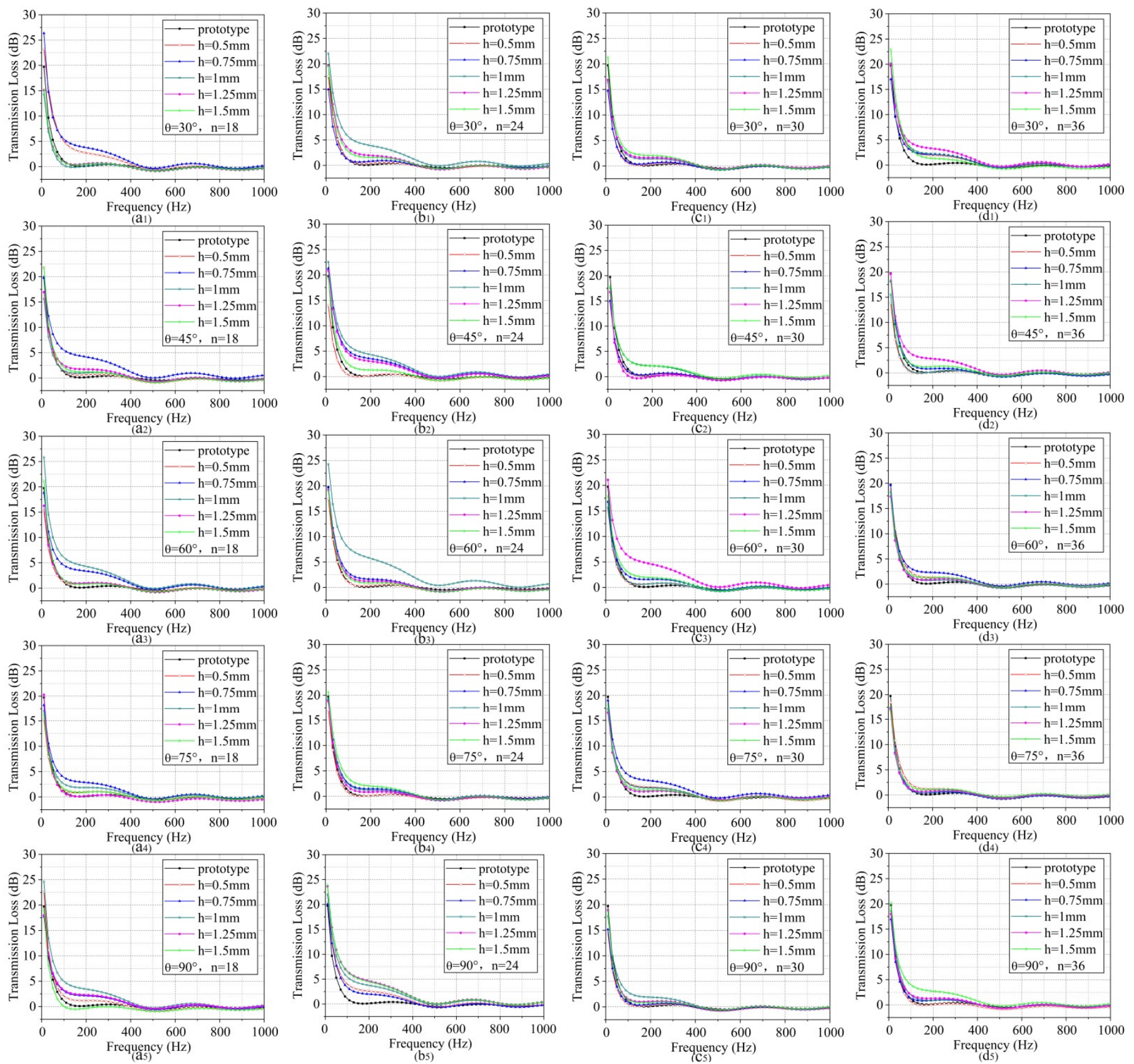

Figure 10. Transmission loss curves at different size parameters: $\left(\mathbf{a}_{1}-\mathbf{a}_{5}\right)$ texture height $\mathrm{h} 0.5-1.5 \mathrm{~mm}$, top angle $\theta 30^{\circ}-90^{\circ}$ and the number of circumferential columns $\mathrm{n}$ is $18,\left(\mathbf{b}_{1}-\mathbf{b}_{5}\right)$ texture height $\mathrm{h}$ $0.5-1.5 \mathrm{~mm}$, top angle $\theta 30^{\circ}-90^{\circ}$ and the number of circumferential columns $\mathrm{n}$ is $24,\left(\mathbf{c}_{1}-\mathbf{c}_{5}\right)$ texture height $\mathrm{h}$ 0.5-1.5 mm, top angle $\theta 30^{\circ}-90^{\circ}$ and the number of circumferential columns $\mathrm{n}$ is $30,\left(\mathbf{d}_{\mathbf{1}}-\mathbf{d}_{\mathbf{5}}\right)$ texture height $\mathrm{h} 0.5-1.5 \mathrm{~mm}$, top angle $\theta 30^{\circ}-90^{\circ}$ and the number of circumferential columns $\mathrm{n}$ is 36 .

As shown in Figure 10( $\left.a_{1}-a_{5}\right)$, within the target frequency range of $10-490 \mathrm{~Hz}$, the transmission loss of the bionic exhaust tailpipes is improved compared to the prototype when the number of circumferential columns is 18 . Figure $10\left(\mathrm{a}_{1}\right)$ shows that the transmission loss $\mathrm{TL}_{0.75 \mathrm{~mm}}>\mathrm{TL}_{0.5 \mathrm{~mm}}>\mathrm{TL}_{1.25 \mathrm{~mm}}>\mathrm{TL}_{1.5 \mathrm{~mm}}>\mathrm{TL}_{1 \mathrm{~mm}}$ as the top angle $\theta$ is $30^{\circ}$. In this paper, $T L_{h} \mathrm{~mm}$ represents the transmission loss at a texture height of $h$ $\mathrm{mm}$. The maximum transmission loss is increased by $6.62 \mathrm{~dB}$, and the minimum transmission loss is increased by $0.128 \mathrm{~dB}$ for a texture height of $0.75 \mathrm{~mm}$. Figure $10\left(\mathrm{a}_{2}\right)$ shows that $\mathrm{TL}_{0.75 \mathrm{~mm}}>\mathrm{TL}_{1.25 \mathrm{~mm}}>\mathrm{TL}_{1.5 \mathrm{~mm}}>\mathrm{TL}_{1 \mathrm{~mm}}>\mathrm{TL}_{0.5 \mathrm{~mm}}$ when the top angle $\theta$ is $45^{\circ}$. The maximum transmission loss is increased by $4.484 \mathrm{~dB}$, and the minimum trans- 
mission loss is increased by $0.172 \mathrm{~dB}$ for a texture height of $0.75 \mathrm{~mm}$. Figure $10\left(\mathrm{a}_{3}\right)$ shows that $\mathrm{TL}_{1 \mathrm{~mm}}>\mathrm{TL}_{0.75 \mathrm{~mm}}>\mathrm{TL}_{1.25 \mathrm{~mm}}>\mathrm{TL}_{1.5 \mathrm{~mm}}>\mathrm{TL}_{0.5 \mathrm{~mm}}$ when the top angle $\theta$ is $60^{\circ}$. The maximum transmission loss is increased by $6.12 \mathrm{~dB}$, and the minimum transmission loss is increased by $0.348 \mathrm{~dB}$ for a texture height of $1 \mathrm{~mm}$. Figure $10\left(\mathrm{a}_{4}\right)$ shows that $\mathrm{TL}_{0.75 \mathrm{~mm}}>\mathrm{TL}_{1 \mathrm{~mm}}>\mathrm{TL}_{1.5 \mathrm{~mm}}>\mathrm{TL}_{0.5 \mathrm{~mm}}>\mathrm{TL}_{1.25 \mathrm{~mm}}$ when the top angle $\theta$ is $75^{\circ}$, the maximum transmission loss is increased by $2.951 \mathrm{~dB}$, and the minimum transmission loss is decreased by $1.5 \mathrm{~dB}$ for the texture height of $0.75 \mathrm{~mm}$. Figure $10\left(\mathrm{a}_{5}\right)$ shows that $\mathrm{TL}_{1 \mathrm{~mm}}>\mathrm{TL}_{1.25 \mathrm{~mm}}>\mathrm{TL}_{0.75 \mathrm{~mm}}>\mathrm{TL}_{0.5 \mathrm{~mm}}>\mathrm{TL}_{1.5 \mathrm{~mm}}$ when the top angle $\theta$ is $90^{\circ}$, the maximum transmission loss is increased by $4.917 \mathrm{~dB}$, and the minimum transmission loss is increased by $0.155 \mathrm{~dB}$ for a texture height of $1 \mathrm{~mm}$. As shown from the transmission loss curves in Figure 10 $\left(a_{1}-a_{5}\right)$, the transmission loss generally increases first and then decreases as the texture height increases under the same top angle $\theta$ and the number of circumferential columns $\mathrm{n}$. The increase in transmission loss is larger near heights of $0.75 \mathrm{~mm}$ and $1 \mathrm{~mm}$. The trend of the transmission loss curve is not obvious as the top angle increases, and the transmission loss is larger when the top angle is near $45^{\circ}, 60^{\circ}$, and $90^{\circ}$.

As shown in Figure $10\left(b_{1}-b_{5}\right)$, the transmission loss is improved compared to the prototype in the target frequency range of $10-490 \mathrm{~Hz}$. Figure $10\left(b_{1}\right)$ shows that the transmission loss is maximum when the top angle $\theta$ is $30^{\circ}$ and the texture height $h$ is $1 \mathrm{~mm}$. The transmission loss maximum is increased by $4.651 \mathrm{~dB}$ and the minimum is increased by $0.356 \mathrm{~dB}$ compared with the prototype. Figure $10\left(\mathrm{~b}_{2}\right)$ shows that the transmission loss is maximum when the top angle $\theta$ is $45^{\circ}$ and the texture height $\mathrm{h}$ is $1 \mathrm{~mm}$. The transmission loss maximum is increased by $5.159 \mathrm{~dB}$ and the minimum is increased by $0.446 \mathrm{~dB}$ at a height of $1 \mathrm{~mm}$ compared with the prototype. Figure $10\left(b_{3}\right)$ shows that the transmission loss reaches a maximum when the top angle $\theta$ is $60^{\circ}$ and the texture height $\mathrm{h}$ is $1 \mathrm{~mm}$. The transmission loss maximum is increased by $6.7 \mathrm{~dB}$ and the minimum is increased by $0.832 \mathrm{~dB}$ compared with the prototype. Figure $10\left(\mathrm{~b}_{4}\right)$ shows that the transmission loss is at maximum when the top angle $\theta$ is $75^{\circ}$, texture height $\mathrm{h}$ is $1.5 \mathrm{~mm}$, transmission loss maximum is increased by $2.927 \mathrm{~dB}$, and minimum is decreased by $0.19 \mathrm{~dB}$ compared with the prototype. Figure $10\left(b_{5}\right)$ shows that the transmission loss is at maximum when the top angle $\theta$ is $90^{\circ}$ and the texture height $\mathrm{h}$ is $1.5 \mathrm{~mm}$. The transmission loss is maximum when it is increased by $5.720 \mathrm{~dB}$ and the minimum when it is increased by $0.539 \mathrm{~dB}$ compared with the prototype. It can be seen from Figure $10\left(b_{1}-b_{5}\right)$ that the transmission loss of the circumferential columns is 24 is improved compared to columns is 18 .

The transmission loss tends to increase first and then decrease as the height increases. The increase in transmission loss is larger near a height of $1 \mathrm{~mm}$. As the top angle increases, the trend of the transmission loss curve is not obvious, and the transmission loss is larger near $45^{\circ}, 60^{\circ}$, and $90^{\circ}$.

From the transmission loss curves in Figure $10\left(c_{1}-c_{5}, d_{1}-d_{5}\right)$, it can be seen that compared with the cases of 18 and 24 circumferential columns, as shown in Figure $10\left(a_{1}-a_{5}, b_{1}-b_{5}\right)$, the transmission loss decreases as the number of circumferential columns increases. The transmission loss is reduced at some frequencies, but the whole transmission loss is improved compared with the prototype.

It can be seen from Figure 10 that within the scope of the study, the number of circumferential columns has a large effect on the transmission loss. As the number of circumferential columns was 24 , the transmission loss improved more obviously. As the height increases, the transmission loss tends to increase first and then decrease. The bionic texture has a larger transmission loss near heights of $0.75 \mathrm{~mm}$ and $1 \mathrm{~mm}$. As the top angle increases, the trend of the transmission loss curve is not obvious. When the top angles are approximately $45^{\circ}$ and $60^{\circ}$, the transmission loss is relatively large.

To research the size parameter influence on transmission loss, bionic tailpipes with differences in the top angle $\theta$, height $h$, and number of circumferential columns $n$ were analyzed. The research suggested that the effect of the top angle on the transmission loss was not obvious. Because of the bionic triangular convex texture of the direction along the tailpipe acoustic wave propagation and airflow direction, the top angle $\theta$ did 
not greatly change the tailpipe acoustic wave propagation. The texture height and the number of circumferential columns played a more significant role in transmission loss. As the texture height was low, the inner surface of the pipe was not much different from the smooth wall surface of the prototype, so the effect of the texture height on the transmission loss was relatively low. As the texture height was high enough, the texture decreased the airflow velocity and reduced the acoustic impedance, so the transmission loss was reduced. Therefore, the texture height should be kept moderate. Meanwhile, the number of circumferential columns of the texture should also be kept moderate. The transmission loss increased as the number of circumferential columns increased. The main reason was that the increase in texture hindered the propagation of sound waves, which increased the acoustic impedance inside the pipe and increased the transmission loss. However, when the number of textures reached a certain level, the transmission loss decreased. The main reason was that the airflow velocity near the pipe wall gradually decreased as the number of circumferential columns $n$ gradually increased, which reduced the acoustic impedance of the bionic exhaust tailpipes and reduced the transmission loss.

\subsection{Structural Parameter Optimization}

From the transmission loss curve of Figure 10, there is a downward trend in transmission loss as the frequency increases, which is the minimum near $490 \mathrm{~Hz}$ in the range of the target frequency between $10 \mathrm{~Hz}$ and $490 \mathrm{~Hz}$. Therefore, the frequency of $490 \mathrm{~Hz}$ is selected as the target optimization frequency. It is obvious from Figure 10 that when the number of circumferential columns is 24 , the transmission loss is at maximum, so the top angle and height need to be optimized for parameters. The transmission loss values corresponding to different heights and top angles at $490 \mathrm{~Hz}$ are summarized in Table 3. The optimization analysis of the neural network algorithm based on genetic algorithm optimization is used to obtain the optimal size parameter and the maximum transmission loss at $490 \mathrm{~Hz}$ so that the transmission loss in the range of $10-490 \mathrm{~Hz}$ is improved as a whole. When the BP neural network optimized by a genetic algorithm is used for size optimization, the population size is 50 , crossover probability selection is 0.3 , mutation probability selection is 0.1 , and evolution algebra is 20. In the process of transmission loss of tractor bionic exhaust tailpipes optimization, since the fitting function has two input parameters and one output parameter, the transmission loss (TL), top angle $\theta$, and texture height $h$ were selected for BP neural network optimization analysis based on genetic algorithm optimization. The variables listed are shown in Table 3. During the optimization process, the input parameters are the bionic texture including top angle $\theta$, texture height $h$, and the maximum transmission loss is the output parameter.

Table 3. Transmission loss at $490 \mathrm{~Hz}$ corresponding to different heights and top angles ( $\theta$ is top angle, $\mathrm{h}$ is texture height, TL is transmission loss).

\begin{tabular}{|c|c|c|c|c|c|c|c|}
\hline Case & TL (dB) & $\theta\left({ }^{\circ}\right)$ & $\mathrm{h}(\mathrm{mm})$ & Case & TL (dB) & $\theta\left({ }^{\circ}\right)$ & $\mathrm{h}(\mathrm{mm})$ \\
\hline 1 & -0.728 & 30 & 0.5 & 14 & 0.346 & 60 & 1.25 \\
\hline 2 & -0.636 & 30 & 0.75 & 15 & -0.824 & 60 & 1.5 \\
\hline 3 & -0.051 & 30 & 1 & 16 & -0.749 & 75 & 0.5 \\
\hline 4 & -0.728 & 30 & 1.25 & 17 & -0.56 & 75 & 0.75 \\
\hline 5 & -0.638 & 30 & 1.5 & 18 & -0.773 & 75 & 1 \\
\hline 6 & -0.766 & 45 & 0.5 & 19 & -0.681 & 75 & 1.25 \\
\hline 7 & -0.17 & 45 & 0.75 & 20 & -0.597 & 75 & 1.5 \\
\hline 8 & 0.039 & 45 & 1 & 21 & -0.548 & 90 & 0.5 \\
\hline 9 & -0.287 & 45 & 1.25 & 22 & -0.636 & 90 & 0.75 \\
\hline 10 & -0.736 & 45 & 1.5 & 23 & -0.036 & 90 & 1 \\
\hline 11 & 0.216 & 60 & 0.5 & 24 & 0.128 & 90 & 1.25 \\
\hline 12 & -0.754 & 60 & 0.75 & 25 & 0.132 & 90 & 1.5 \\
\hline 13 & 0.425 & 60 & 1 & & & & \\
\hline
\end{tabular}


As shown in Figure 11, under different top angles and heights before and after optimization, the trend of the corresponding transmission loss remains the same. The theoretical value of the optimal parameter of the bionic triangle texture was obtained after optimization. When the top angle is $61^{\circ}$ and the texture height is $0.95 \mathrm{~mm}$, the transmission loss corresponding to $490 \mathrm{~Hz}$ is the maximum. The air cavity of bionic exhaust tailpipes with the structural parameters was modeled in three dimensions, and an acoustic simulation was conducted using LMS Virtual Lab acoustic simulation software. Figure 12 shows the comparison of the transmission loss curves of the prototype and the optimized bionic exhaust tailpipe. The increased value represents the difference between the transmission loss of the optimized bionic tailpipe and the prototype tailpipe. In the target frequency range, the bionic exhaust tailpipe transmission loss improved by $1.009 \mathrm{~dB}$ to $9.62 \mathrm{~dB}$ compared with the prototype after optimization. The transmission loss increased by $9.62 \mathrm{~dB}$ at $30 \mathrm{~Hz}$ and increased by $1.009 \mathrm{~dB}$ at $490 \mathrm{~Hz}$. From the beginning of $10 \mathrm{~Hz}$, the increment value increases, and the increment value was at maximum at $30 \mathrm{~Hz}$ and then gradually decreases above $30 \mathrm{~Hz}$. The transmission loss was larger and the noise reduction performance was better when the frequency was between $10 \mathrm{~Hz}$ and $490 \mathrm{~Hz}$, which indicates that the structure optimization was suitable for improving low- and medium-frequency noise reduction performance and was suitable for the issuing of tractor exhaust tailpipe reduction noise.

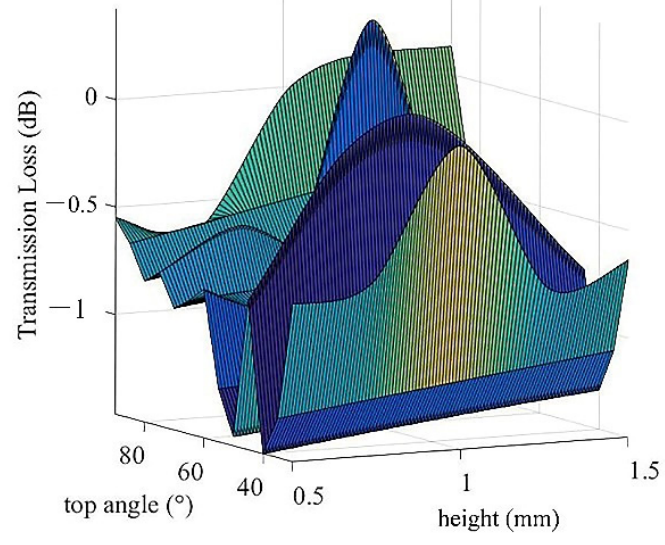

(a)

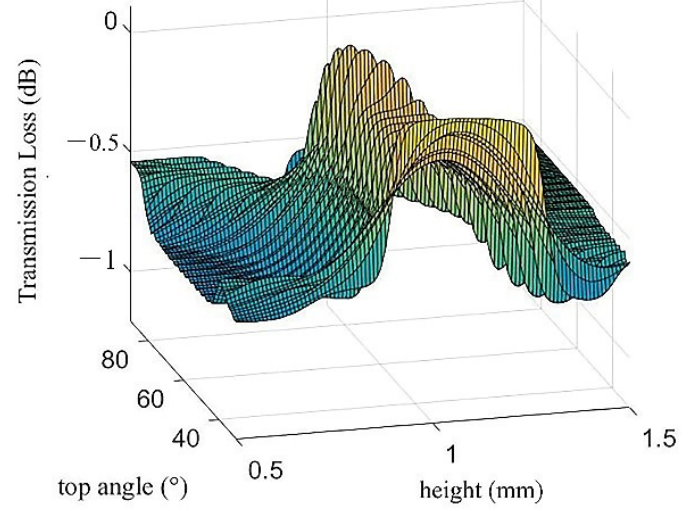

(b)

Figure 11. Relation between texture heights, top angles, and corresponding transmission loss (a) before optimization, (b) after optimization.

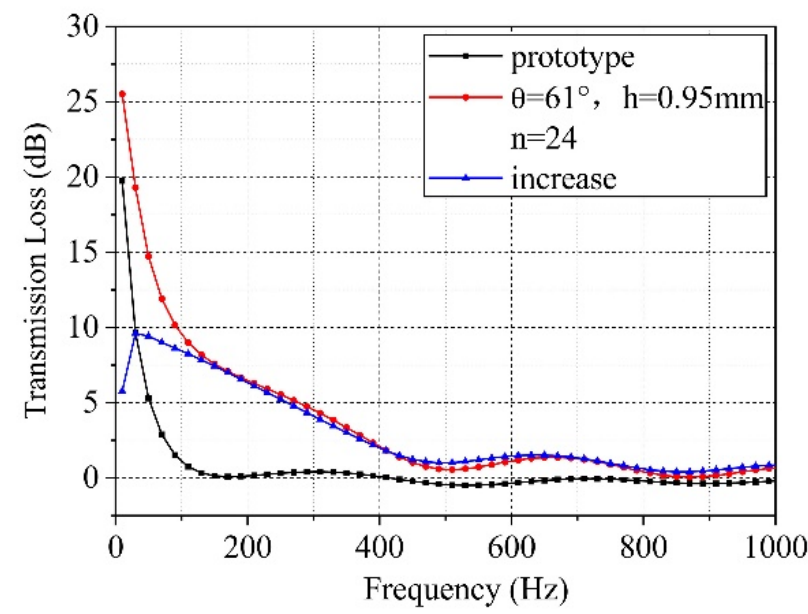

Figure 12. Comparison between prototype and optimized bionic exhaust tailpipe transmission loss curves. 


\subsection{Physical Mechanism of Noise Reduction in Bionic Exhaust Tailpipe}

To study the noise reduction mechanism of the bionic tailpipe better, the pressure nephogram, velocity amplitude nephogram, surface friction coefficient nephogram, and vorticity distribution nephogram were analyzed, as shown in Figures 13-17. As seen from Table 2, when the inlet velocity was $50 \mathrm{~m} / \mathrm{s}$, the noise reduction effect was better than those of other speed conditions. Therefore, the flow field under this inlet velocity condition was compared with the prototype tailpipe to study the noise reduction mechanism. Figure 13 shows a pressure nephogram of the prototype and bionic tailpipe. The wall pressure of the bionic tailpipe was less than that of the prototype tailpipe. The results show that the bionic texture reduced the wall air pressure, increased the stability of airflow near the wall, and weakened the generation of turbulence. This had a positive effect on the flow stability near the whole pipe wall, which reduces the aerodynamic noise of the exhaust tailpipe. As seen from Figure 14, the comparison nephogram of the vertical flow section velocity amplitude between the prototype and bionic tailpipe and the cross-section was at $300 \mathrm{~mm}$ in the $z$-axis direction. The velocity amplitude near the texture of the bionic tailpipe was smaller than that at the same position as the prototype tailpipe. The results showed that the bionic texture can reduce the velocity amplitude near the wall and weaken the turbulence near the wall. As shown in Figure 15, the friction coefficient of the surface near the inlet of the pipeline was significantly reduced through numerical simulation. Therefore, the bionic texture reduces the resistance between the airflow and the wall of the bionic tailpipe. As shown in Table 4, the drag reduction effect under different conditions was analyzed, and the drag reduction rate of the smooth surface model and nonsmooth surface model was obtained under different conditions. The drag reduction rate improved with increasing inlet velocity.

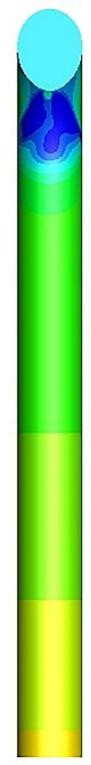

Pressure $(\mathrm{Pa})$

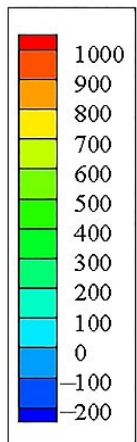

(a)

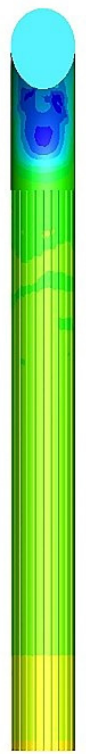

Pressure $(\mathrm{Pa})$

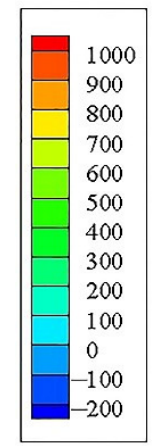

(b)

Figure 13. Comparison of pressure nephogram: (a) prototype and (b) bionic tailpipe.

A comparison of the vorticity distribution nephogram between the prototype and bionic tailpipe is shown in Figure 16. The research suggests that the vorticity amplitude in most areas of the bionic tailpipe wall is reduced compared with the prototype tailpipe. The bionic texture can make the airflow near the tailpipe wall more stable, suppress turbulence bursts, and reduce aerodynamic noise. An X-vorticity nephogram of the vertical flow section is shown in Figure $17(z=300 \mathrm{~mm})$. There are obvious secondary vortices at the tip of the bionic texture. The secondary vortex is the fundamental reason for drag reduction. The rotation direction of the secondary vortex is opposite to that of the large vortex, which weakens the large eddy and weakens the turbulent flow. The two opposite vortices that appear near the bionic texture undermine each other, which makes the airflow around the 
wall of the bionic tailpipe more stable and reduces the generation of aerodynamic noise. At the same time, the interaction between the secondary vortex pair and the reverse vortex pair weakens the reverse vortex pair, suppresses the turbulence burst, reduces the energy dissipation, and produces drag reduction and noise reduction.

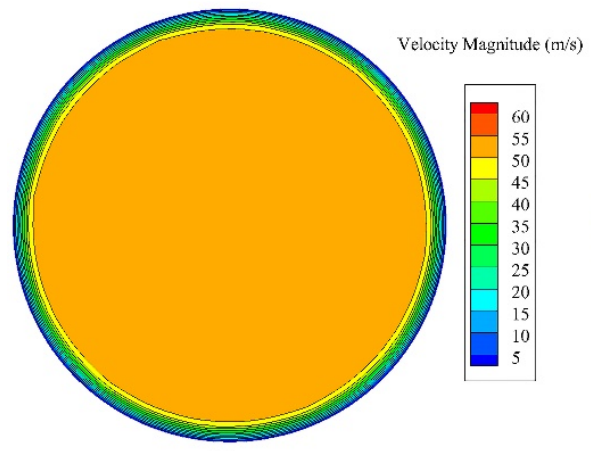

(a)

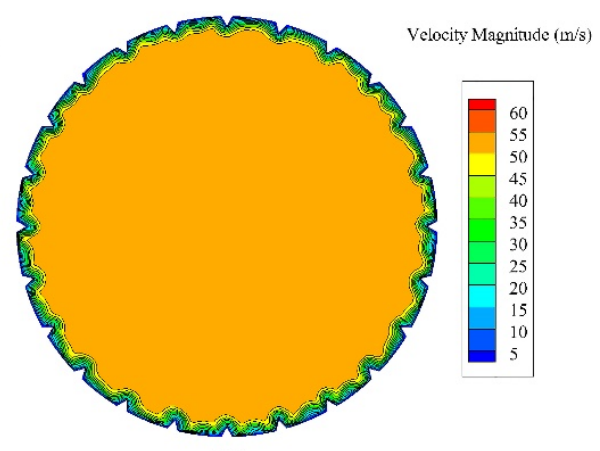

(b)

Figure 14. Vertical flow direction section velocity amplitude nephogram: (a) prototype and (b) bionic tailpipe.

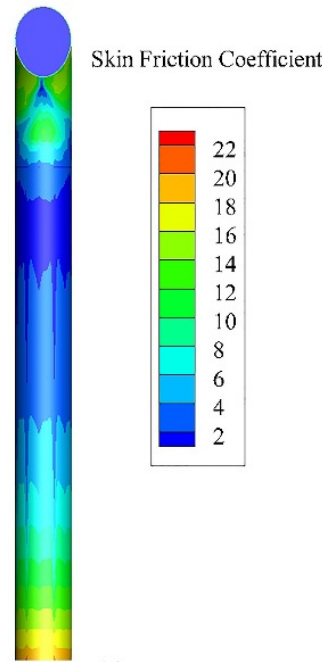

(a)

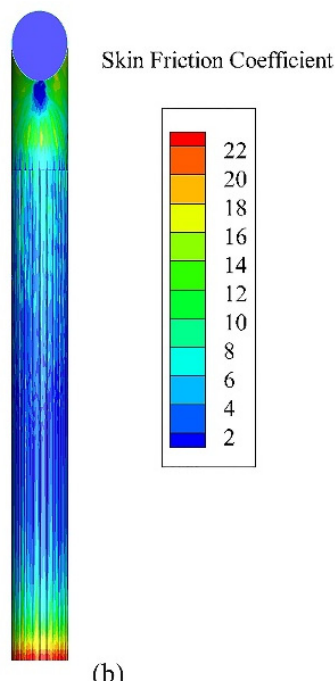

(b)

Figure 15. Surface friction coefficient nephogram: (a) prototype and (b) bionic tailpipe.

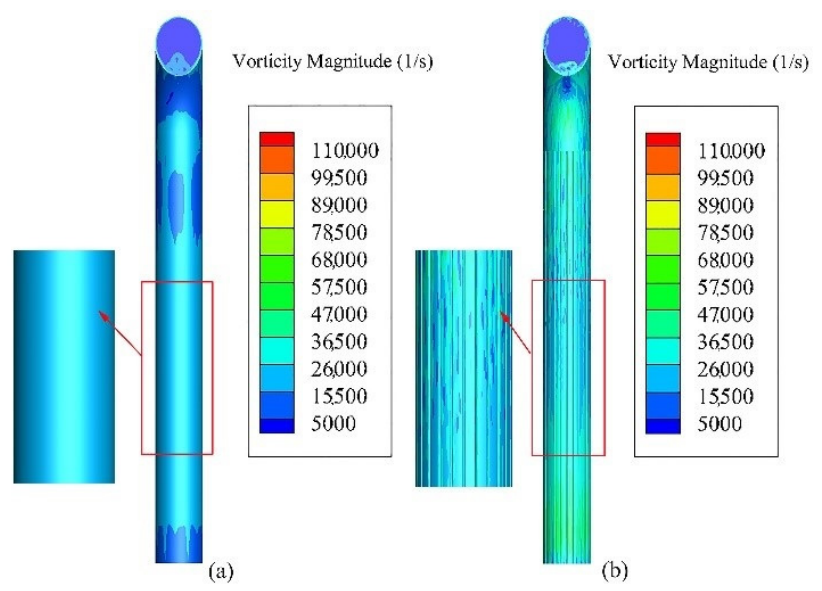

Figure 16. Vorticity amplitude nephogram (a) prototype and (b) bionic tailpipe. 


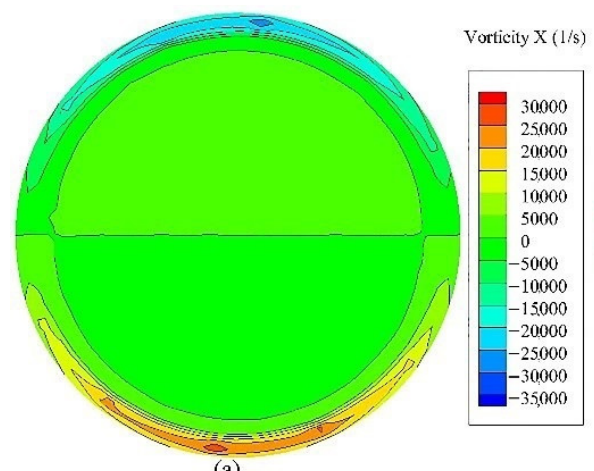

(a)

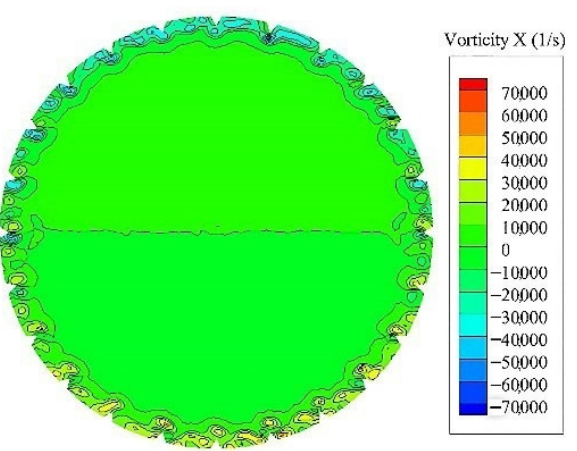

(b)

Figure 17. X-vorticity nephogram of vertical flow section in (a) prototype and (b) bionic tailpipe.

Table 4. The surface friction coefficient of the tailpipe wall at different inlet velocities.

\begin{tabular}{cccc}
\hline Velocities $\mathbf{( m} / \mathbf{s})$ & Prototype & Bionic Tailpipes & Drag Reduction \\
\hline 40 & 4.219 & 3.901 & $7.54 \%$ \\
50 & 6.313 & 5.650 & $10.50 \%$ \\
60 & 8.859 & 7.641 & $13.75 \%$ \\
70 & 11.752 & 9.905 & $15.72 \%$ \\
\hline
\end{tabular}

Figures 18 and 19 show the sound pressure level amplitude nephogram (a), velocity amplitude nephogram (b), and velocity vector diagram (c) of the prototype and optimized bionic exhaust tailpipe of the tractor at $490 \mathrm{~Hz}$, respectively, to better study the vibration noise reduction mechanism of the bionic tailpipe. As shown in Figures 18a and 19a, the sound pressure level amplitude of the prototype exhaust tailpipe at the inlet was concentrated at $146 \mathrm{~dB}$, and the sound pressure level amplitude at the outlet was concentrated at $144 \mathrm{~dB}$. The sound pressure level amplitude of the whole tailpipe was mainly between $141 \mathrm{~dB}$ and $144 \mathrm{~dB}$. The sound pressure level amplitude of the bionic exhaust tailpipe concentrates at $146 \mathrm{~dB}$ at the inlet and $143 \mathrm{~dB}$ at the outlet. The sound pressure level amplitude of the whole tailpipe was mainly between $139 \mathrm{~dB}$ and $143 \mathrm{~dB}$. The bionic texture plays a role in reducing the sound pressure level amplitude inside the tailpipe. From the velocity amplitude nephogram in Figures $18 \mathrm{~b}$ and $19 \mathrm{~b}$, the velocity amplitude at the inlet of the prototype tailpipe was mainly between $3.22 \mathrm{~m} / \mathrm{s}$ and $3.57 \mathrm{~m} / \mathrm{s}$, and the outlet was mainly between $1.81 \mathrm{~m} / \mathrm{s}$ and $2.51 \mathrm{~m} / \mathrm{s}$. The velocity amplitude of the entire tailpipe was mainly between $0.752 \mathrm{~m} / \mathrm{s}$ and $1.46 \mathrm{~m} / \mathrm{s}$. However, the velocity amplitude of the entire pipeline of the bionic exhaust tailpipe was concentrated at $0.37 \mathrm{~m} / \mathrm{s}$. This shows that the bionic triangle texture reduced the velocity amplitude, reducing the sound pressure level.

From the velocity vector diagrams in Figures $18 \mathrm{c}$ and $19 \mathrm{c}$, the velocity at the inlet of the prototype tailpipe was $0.644 \mathrm{~m} / \mathrm{s}$, and the direction was the same as the exhaust direction. The velocity at the outlet was between $1.61 \mathrm{~m} / \mathrm{s}$ and $1.93 \mathrm{~m} / \mathrm{s}$, and the direction was opposite to the exhaust. The minimum velocity was in the middle and lower parts of the prototype exhaust tailpipe, and the speed was $2.14 \times 10^{-3} \mathrm{~m} / \mathrm{s}$. The velocity of the bionic tailpipe inlet was mainly between $0.426 \mathrm{~m} / \mathrm{s}$ and $0.852 \mathrm{~m} / \mathrm{s}$. The direction was the same as the exhaust direction, and the speed at the outlet was between $2.13 \mathrm{~m} / \mathrm{s}$ and $4.26 \mathrm{~m} / \mathrm{s}$, while the direction was opposite to the exhaust direction. The minimum velocity was in the middle and lower parts of the tailpipe, and the speed is $4.8 \times 10^{-5} \mathrm{~m} / \mathrm{s}$. From the diagrams of the entire tailpipe speed vector, the internal velocity of the prototype tailpipe was between $2.14 \times 10^{-3} \mathrm{~m} / \mathrm{s}$ and $0.964 \mathrm{~m} / \mathrm{s}$, and the internal speed of the bionic tailpipe was between $4.8 \times 10^{-5} \mathrm{~m} / \mathrm{s}$ and $0.426 \mathrm{~m} / \mathrm{s}$. The maximum reverse velocity at the outlet of the prototype tailpipe was $3.21 \mathrm{~m} / \mathrm{s}$. However, the reverse velocity of the bionic tailpipe reached $4.26 \mathrm{~m} / \mathrm{s}$, which had a greater hedging effect on sound wave propagation along the pipeline, reduced the sound velocity in the pipeline, and reduced the internal sound pressure of the pipe. 


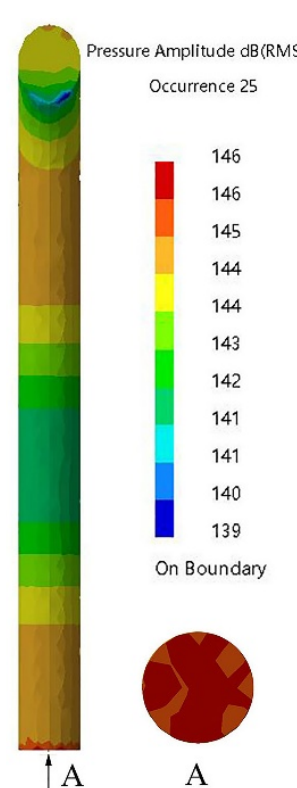

(a)

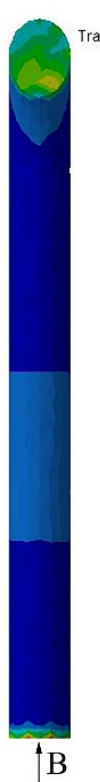

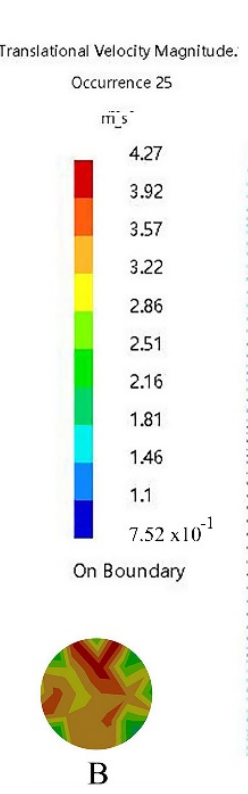

(b)

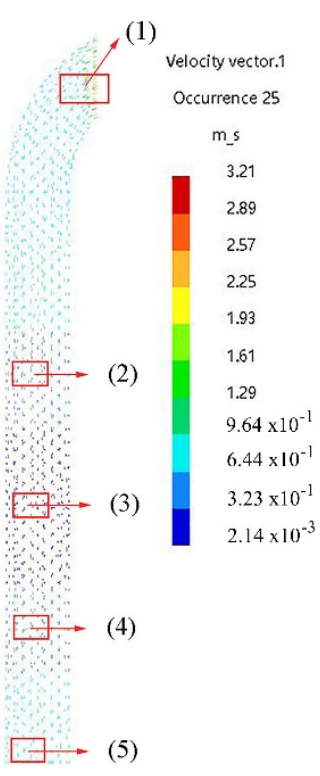

(1)

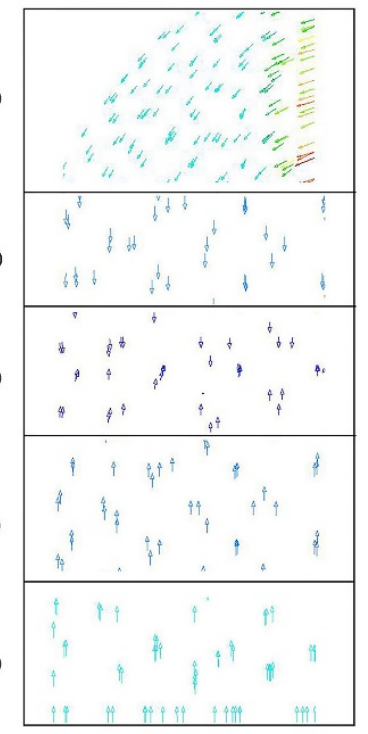

(c)

Figure 18. Vorticity amplitude nephogram (a) prototype (b) bionic tailpipe. Prototype: (a) sound pressure level amplitude nephogram, (b) velocity amplitude nephogram, and (c) velocity vector diagram.
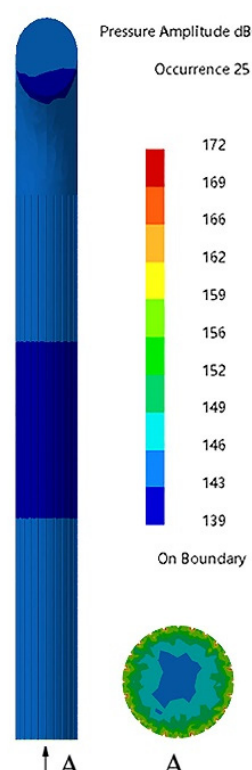

A

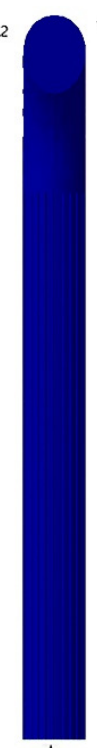

$\uparrow \mathrm{B}$

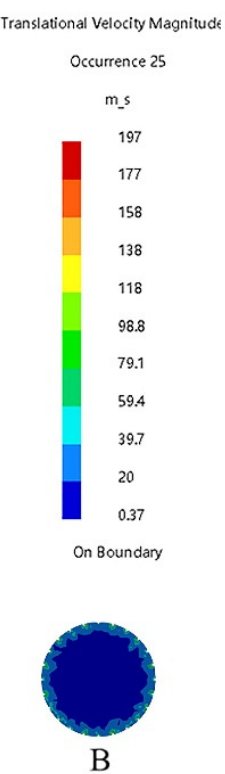

(b)

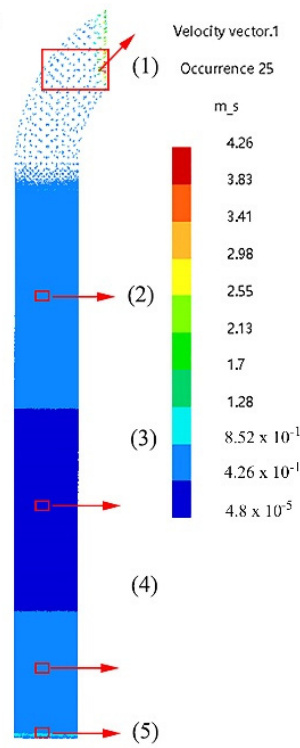

4.26

3.83

2.41

2.55

2.13

(3)

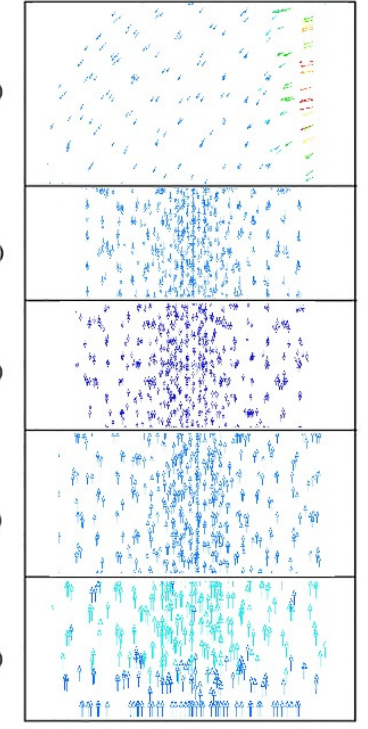

(c)

Figure 19. Optimized bionic exhaust tailpipe: (a) sound pressure level amplitude nephogram, (b) velocity amplitude nephogram, and (c) velocity vector diagram.

\subsection{Experience Results and Analysis}

In this section, the results of the insertion loss tests are analyzed. The analysis focused on the noise reduction effect of the application of bionic textures in the inner surface of tailpipes. As shown in Figure 20( $\left.a_{1}\right)$ and Figure $21\left(a_{1}\right)$, the top angle of the bionic texture was $45^{\circ}$, and the texture height was $0.75 \mathrm{~mm}$. When the frequency is $100-2000 \mathrm{~Hz}$, the maximum insertion loss could reach $4.351 \mathrm{~dB}$ at $1000 \mathrm{~Hz}$. In Figure 20( $\left.\mathrm{b}_{1}\right)$ and Figure 21( $\left.\mathrm{b}_{1}\right)$, the top angle of the bionic texture was $45^{\circ}$ and the texture height is $1 \mathrm{~mm}$. Except for the negative value at $50 \mathrm{~Hz}$, the average insertion loss at other frequency bands was $3.142 \mathrm{~dB}$. In Figure $20\left(\mathrm{c}_{1}\right)$ and Figure $21\left(\mathrm{c}_{1}\right)$, the top angle was $45^{\circ}$ and the texture height 
was $1.25 \mathrm{~mm}$. The insertion loss was positive throughout the test frequency range, with an average insertion loss of $1.338 \mathrm{~dB}$. In Figure $20\left(\mathrm{a}_{2}\right)$ and Figure $21\left(\mathrm{a}_{2}\right)$, the top angle is $60^{\circ}$, and the texture height is $0.75 \mathrm{~mm}$. The maximum insertion loss was $2.372 \mathrm{~dB}$ at $630 \mathrm{~Hz}$. In Figure 20( $\left.b_{2}\right)$ and Figure 21( $\left.b_{2}\right)$, the top angle was $60^{\circ}$ and the texture height is $1 \mathrm{~mm}$. The insertion loss was greater than $1 \mathrm{~dB}$ at most frequencies from $63 \mathrm{~Hz}$ to $1600 \mathrm{~Hz}$. The insertion loss was $4.374 \mathrm{~dB}$ at $1000 \mathrm{~Hz}$. In Figure 20( $\left.\mathrm{c}_{2}\right)$ and Figure $21\left(\mathrm{c}_{2}\right)$, the top angle is $60^{\circ}$ and the texture height was $1.25 \mathrm{~mm}$. Between $63 \mathrm{~Hz}$ to $2000 \mathrm{~Hz}$, the maximum insertion loss was $3.887 \mathrm{~dB}$ at $2000 \mathrm{~Hz}$. In Figure 20( $\left.\mathrm{a}_{3}\right)$ and Figure 21( $\left.\mathrm{a}_{3}\right)$, the top angle was $75^{\circ}$, the texture height was $0.75 \mathrm{~mm}$, and the insertion loss was about $2 \mathrm{~dB}$ at most frequencies. The maximum insertion loss at $2000 \mathrm{~Hz}$ was $5.182 \mathrm{~dB}$. In Figure 20( $\left.b_{3}\right)$ and Figure 21( $\left.\mathrm{b}_{3}\right)$, the top angle is $75^{\circ}$ and the texture height was $1 \mathrm{~mm}$. Between $100 \mathrm{~Hz}$ and $630 \mathrm{~Hz}$, insertion loss was about $2 \mathrm{~dB}$ at some of these frequencies and $3.274 \mathrm{~dB}$ at $630 \mathrm{~Hz}$. In Figure 20( $\left.\mathrm{c}_{3}\right)$ and Figure 21( $\left.\mathrm{c}_{3}\right)$, the top angle was $75^{\circ}$, the texture height was $1.25 \mathrm{~mm}$, and the average insertion loss of other frequency bands was $1.918 \mathrm{~dB}$ except for the negative value of $50 \mathrm{~Hz}$.

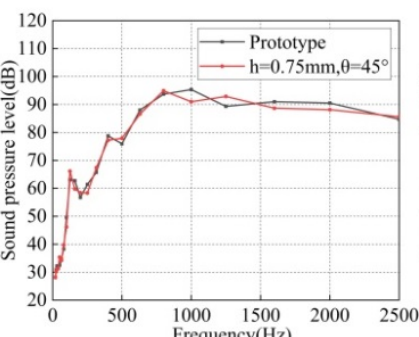

(a1)

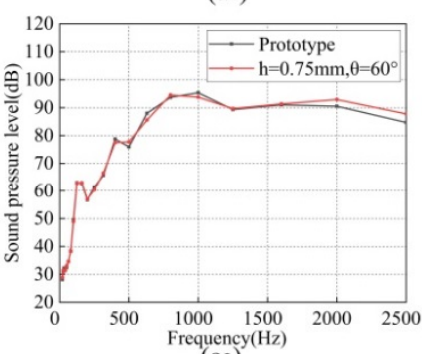

(a2)

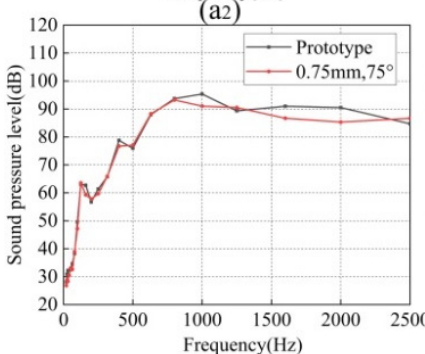

(a3)

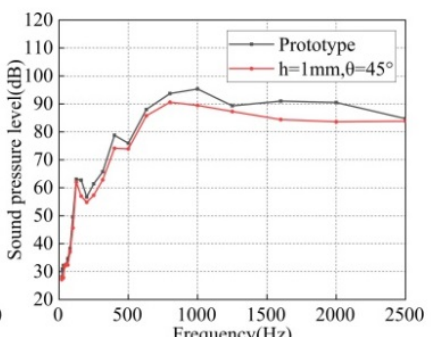

(b1)

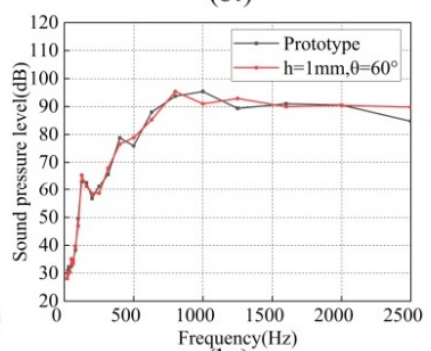

(b2)

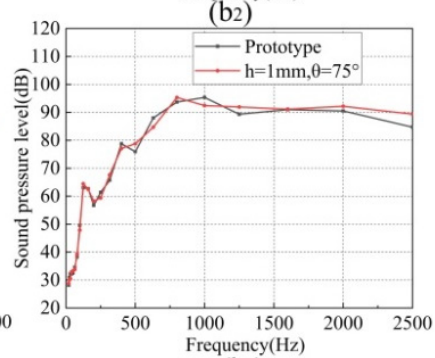

(b3)

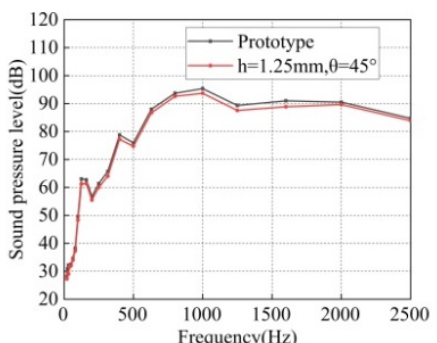

(c1)

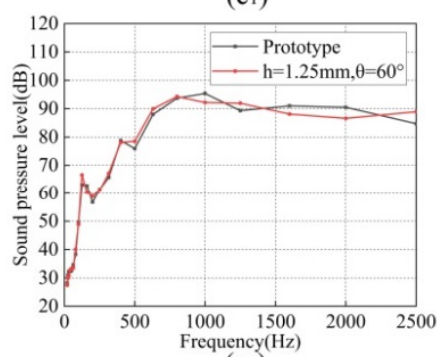

(c2)

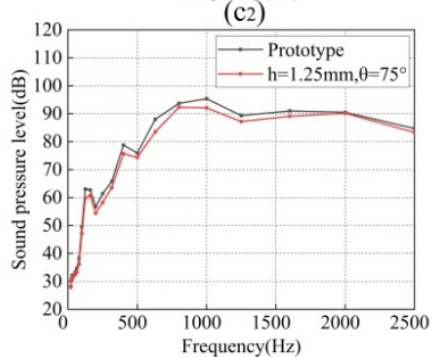

(c3)

Figure 20. Sound pressure level curves at different size parameters: $\left(\mathbf{a}_{1}-\mathbf{a}_{3}\right)$ texture height $\mathrm{h} 0.75 \mathrm{~mm}$ and top angle $\theta 45^{\circ}-75^{\circ},\left(\mathbf{b}_{1}-\mathbf{b}_{3}\right)$ texture height $\mathrm{h} 1 \mathrm{~mm}$ and top angle $\theta 45^{\circ}-75^{\circ},\left(\mathbf{c}_{1}-\mathbf{c}_{3}\right)$ texture height $\mathrm{h} 1.25 \mathrm{~mm}$ and top angle $\theta 45^{\circ}-75^{\circ}$.

Figures 20 and 21 show the curves of the sound pressure level and insertion loss measured in the experiment. The sound pressure level of the bionic exhaust tailpipes decreased to different degrees in most frequency bands compared with the prototype exhaust tailpipe. On the whole, the noise reduction effect was better when the top angle was approximately $60^{\circ}$, which was consistent with the simulation results. In the range of $45^{\circ}$ and $60^{\circ}$, the insertion loss tends to increase first and then decrease with increasing texture height at the same top angle, and the effect of noise reduction was better when the texture height was $1 \mathrm{~mm}$, which was also consistent with the simulation results. When the top angle reached $75^{\circ}$, the effect of different texture heights on the insertion loss was less than that of other angles, and the change trend was not obvious, which was slightly 
different from the simulation results. The disparity was mainly due to the limitation of experimental conditions, and the influence of airflow velocity was not considered in the experiment.
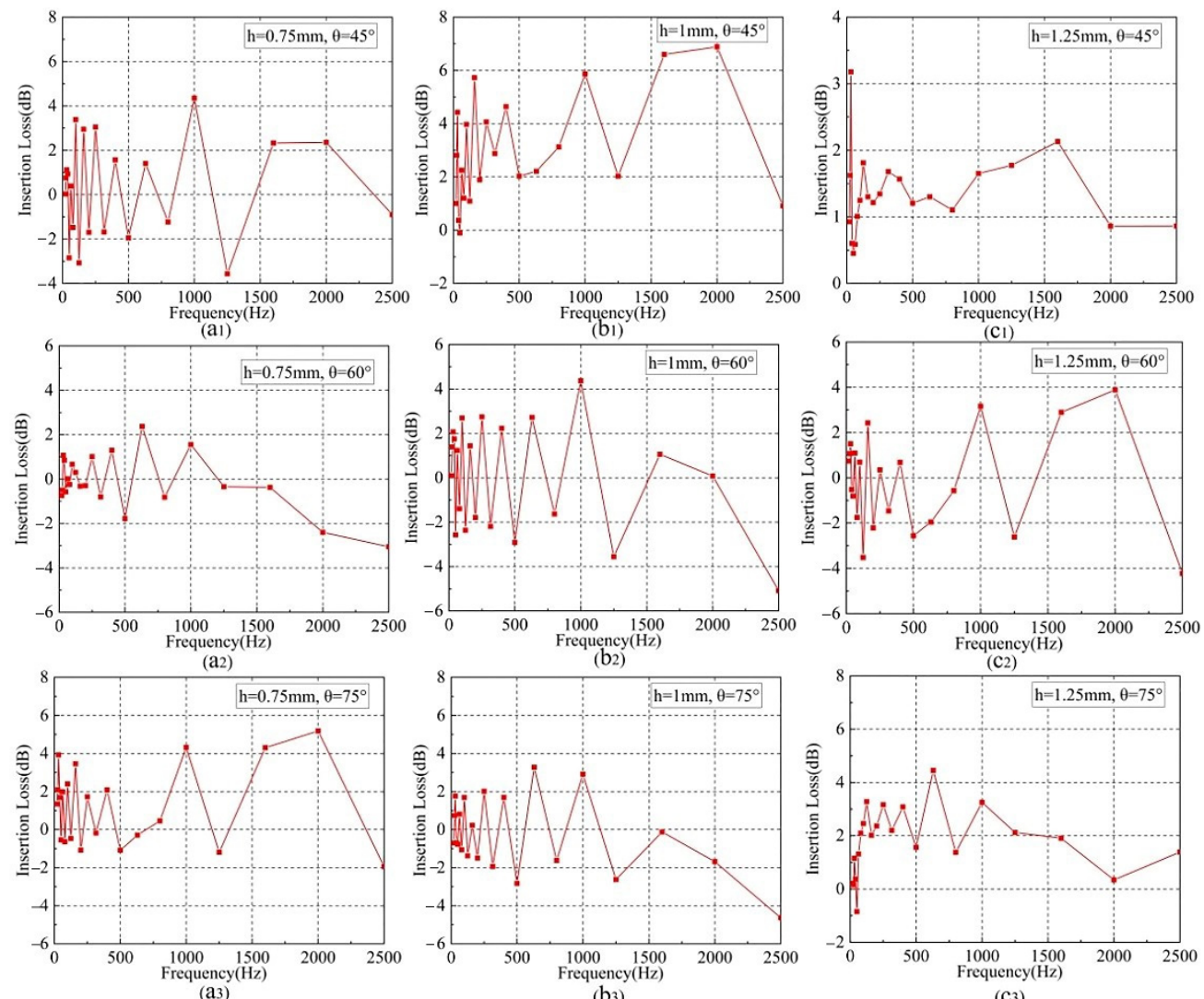

(b3)

(C3)

Figure 21. Insertion loss curves at different size parameters: $\left(\mathbf{a}_{1}-\mathbf{a}_{3}\right)$ texture height $\mathrm{h} 0.75 \mathrm{~mm}$ and top angle $\theta 45^{\circ}-75^{\circ},\left(\mathbf{b}_{1}-\mathbf{b}_{3}\right)$ texture height $\mathrm{h} 1 \mathrm{~mm}$ and top angle $\theta 45^{\circ}-75^{\circ},\left(\mathbf{c}_{1}-\mathbf{c}_{3}\right)$ texture height $\mathrm{h}$ $1.25 \mathrm{~mm}$ and top angle $\theta 45^{\circ}-75^{\circ}$.

\section{Conclusions}

By imitating the surface of a shark with triangular grooves, a bionic triangular convex texture was added to the inner surface of an exhaust tailpipe to address noise reduction. The bionic tailpipes produced different degrees of noise reduction on aeroacoustics and transmission loss compared with a prototype exhaust tailpipe.

From the perspective of aeroacoustics, the total sound pressure level changes the most at $50 \mathrm{~m} / \mathrm{s}$, reaching $2.560 \mathrm{~dB}$ compared with other speed conditions. At this speed, the bionic texture shows the best noise reduction, corresponding to a Reynolds number of $1.51 \times 10^{5}$. The influence of texture circumferential column number $\mathrm{n}$ was the most significant. Texture height $h$ influenced noise reduction, and top angle $\theta$ had no obvious effect on the noise reduction within the research range.

To study vibration noise reduction, bionic triangular convex textures with different size parameters were simulated and analyzed. The research showed that the transmission loss was relatively large, the reduction performance was relatively good, the number of circumferential columns was 24 , the top angles were $45^{\circ}$ and $60^{\circ}$, and the texture heights were $0.75 \mathrm{~mm}$ and $1 \mathrm{~mm}$. The BP neural network algorithm based on GA optimization was used to optimize the structure parameters. The corresponding transmission loss reached the maximum when the top angle $\theta$ was $61^{\circ}$ and the texture height $h$ was $0.95 \mathrm{~mm}$.

By analyzing the noise reduction mechanism of aeroacoustics, the bionic texture can reduce the air pressure and velocity amplitude near the wall of the bionic tailpipe. The secondary vortex appears near the bionic texture, reducing aerodynamic drag and 
aeroacoustics. By analyzing the noise reduction mechanism of vibration noise, the bionic triangular convex texture reduced the velocity amplitude of the exhaust tailpipe and reduced the sound pressure inside the exhaust tailpipe of the tractor.

The experimental results demonstrated that the bionic exhaust tailpipes reduced the noise. On the whole, the noise reduction effect is better when the top angle is $60^{\circ}$ and the texture height is $1 \mathrm{~mm}$, which is consistent with the simulation results.

It is feasible to apply the bionic triangle texture to the tractor tailpipe. The total sound pressure level can be reduced and the transmission loss can be increased, so a certain noise reduction effect can be achieved by adding a bionic triangular convex texture inside the tailpipe without changing the overall size parameters of the prototype tailpipe.

Author Contributions: Z.Z., Z.H. and Q.W. conceived the conceptualization; S.Z. and T.S. designed the experiments; Z.Z., T.S. and T.L. performed the experiments; Z.H. and T.S. analyzed the data; Z.Z. and Z.H. wrote the paper. All authors have read and agreed to the published version of the manuscript.

Funding: This work was funded by the National Key Research and Development Program of China in the 13th Five-year Plan Period (2016YFD0701102) and the science and technology projects in Jilin Province Education Department (No. JJKH20211064KJ).

Institutional Review Board Statement: Not applicable.

Informed Consent Statement: Not applicable.

Data Availability Statement: Not applicable.

Conflicts of Interest: The authors declare no conflict of interest.

\section{References}

1. Fu, J.; Xu, M.H.; Wei, Z. Effects of structural parameters on transmission loss of diesel engine muffler and analysis of prominent structural parameters. Appl. Acoust. 2021, 173, 107686. [CrossRef]

2. Fu, J.; Wei, Z.; Xu, M.H. Study on the influence of structure factors of diesel engine exhaust purification muffler on transmission loss in different frequency bands. Appl. Acoust. 2021, 180, 108147. [CrossRef]

3. Cambonie, T.; Mbailassem, F.; Gourdon, E. Bending a quarter wavelength resonator: Curvature effects on sound absorption properties. Appl. Acoust. 2018, 131, 87-102. [CrossRef]

4. Xue, F.; Sun, B.B. Experimental study on the comprehensive performance of the application of U-shaped corrugated pipes into reactive mufflers. Appl. Acoust. 2018, 141, 362-370. [CrossRef]

5. Segin, E.; Arslan, H.; Birgoren, B. A statistical design optimization study of a multi-chamber reactive type silencer using simplex centroid mixture design. J. Low Freq. Noise Vib. Act. Control 2021, 40, 623-638. [CrossRef]

6. Sagar, V.; Munjal, M.L. Analysis and design guidelines for fork muffler with H-connection. Appl. Acoust. 2017, 125, 49-58. [CrossRef]

7. Zhu, Y.W.; Zhu, F.W.; Zhang, Y.S.; Wei, Q.G. The research onsemi-active muffler device of controlling the exhaust pipe's low-frequency noise. Appl. Acoust. 2017, 116, 9-13. [CrossRef]

8. Mimani, A.; Munjal, M.L. Design of Reactive Rectangular Expansion Chambers for Broadband Acoustic Attenuation Performance based on Optimal Port Location. Acoust. Aust. 2016, 44, 299-323. [CrossRef]

9. Meriç, C.; Erol, H.; Özkan, A. Design and application of a compact helical air intake system resonator for broadband noise control. Appl. Acoust. 2018, 131, 103-111. [CrossRef]

10. Lu, C.H.; Chen, W.; Liu, Z.; Du, S.Z.; Zhu, Y.W. Pilot study on compact wideband micro-perforated muffler with a serial-parallel coupling mode. Appl. Acoust. 2019, 148, 141-150. [CrossRef]

11. Yasuda, T.; Wu, C.; Nakagawa, N.; Nagamura, K. Studies on an automobile muffler with the acoustic characteristic of low-pass filter and Helmholtz resonator. Appl. Acoust. 2013, 74, 49-57. [CrossRef]

12. Xiang, L.Y.; Zuo, S.G.; Wu, X.D.; Liu, J.F. Study of multi-chamber micro-perforated muffler with adjustable transmission loss. Appl. Acoust 2017, 122, 35-40. [CrossRef]

13. Zhang, Y.A.; Wu, P.; Ma, Y.H.; Su, H.; Xue, J. Analysis on acoustic performance and flow field in the split-stream rushing muffler unit. J. Sound Vib. 2018, 430, 185-195. [CrossRef]

14. Li, S.X.; Hou, J.J.; Pan, W.L.; Wang, Z.H.; Kang, Y.X. Study on Aerodynamic Noise Numerical Simulation and Characteristics of Safety Valve Based on Dipole and Quadrupole. Acoust. Aust. 2020, 48, 441-454. [CrossRef]

15. Tu, J.; Gan, L.; Ma, S.; Zhang, H. Flow Noise Characteristics Analysis of Underwater High-Speed Vehicle Based on LES/FW-H Coupling Model. Acoust. Aust. 2019, 47, 91-104. [CrossRef]

16. Yang, L.; Chu, Z.G.; Zhao, D. Analysis and Control for the Intake Noise of a Vehicle. Acoust. Aust. 2018, 46, 259-267. [CrossRef] 
17. Gruschka, H.D.; Borchers, I.U.; Coble, J.G. Aerodynamic Noise produced by a Gliding Owl. Nature 1971, 233, 409-411. [CrossRef]

18. Walsh, M.J. Riblets as Viscous Drag Reduction Technique. AIAA J. 1983, 21, 485-486. [CrossRef]

19. Bachmann, T.; Wagner, H. The three-dimensional shape of serrations at barn owl wings towards a typical natural serration as a role model for biomimetic applications. J. Anat. 2011, 219, 192-202. [CrossRef]

20. Wang, J.; Zhang, C.C.; Wu, Z.Y.; Wharton, J.; Ren, L.Q. Numerical study on reduction of aerodynamic noise around an airfoil with biomimetic structures. J. Sound Vib. 2017, 394, 46-58. [CrossRef]

21. Zhang, Z.J.; Liu, L.J.; Bing, Z. Bionic Exhaust Tail Pipe of Tractor. CN105484844A, 13 April 2016. Available online: https: //patents.google.com/patent/CN105484844A/en (accessed on 6 October 2021).

22. Zhang, Z.J.; Hou, Z.H.; Xin, X.J. Noise reduction of a tractor exhaust tailpipe with bionic convex textures. Noise Control Eng. J. 2019, 67, 363-372. [CrossRef]

23. Roger, M.; Schram, C.; De Santana, L. Reduction of Airfoil Turbulence-Impingement Noise by Means of Leading-Edge Serrations and/or Porous Material. In Proceedings of the 19th AIAA/CEAS Aeroacoustics Conference, Berlin, Germany, $27-29$ May 2013. [CrossRef]

24. Chong, T.P.; Joseph, P.F.; Gruber, M. Airfoil self noise reduction by non-flat plate type trailing edge serrations. Appl. Acoust. 2013, 74, 607-613. [CrossRef]

25. Wang, M.H.; Liu, X.M. Numerical investigation of aerodynamic and acoustic characteristics of bionic airfoils inspired by bird wing. Proc. Inst. Mech. Eng. Part G J. Aerosp. Eng. 2018, 233, 4004-4016. [CrossRef]

26. Liu, X.M.; Zhao, J.; Li, D. Noise reduction mechanism of single-arc bionic blade with wave shape leading edge coupled with serrated trailing edge. J. Xi'an Jiaotong 2015, 49, 1-10. [CrossRef]

27. Li, D.; Liu, X.M. A comparative study on aerodynamic performance and noise characteristics of two kinds of long-eared owl wing models. J. Mech. Sci. Technol. 2017, 31, 3821-3830. [CrossRef]

28. Liu, Y.Y.; Li, Y.Q.; Ding, Z.Y. Numerical simulation on the impact of the bionic structure on aerodynamic noises of sidewindow regions in vehicles. J. Vibroeng. 2018, 20, 1257-1271. [CrossRef]

29. Dean, B.; Bhushan, B. Shark-skin surfaces for fluid-drag reduction in turbulent flow: A review. Philos. Trans. R. Soc. A 2010, 368, 4775-4806. [CrossRef]

30. Shi, L.; Zhang, C.C.; Wang, J.; Ren, L.Q. Numerical Simulation of the Effect of Bionic Serrated Structures on the Aerodynamic Noise of a Circular Cylinder. J. Bionic. Eng. 2012, 9, 91-98. [CrossRef]

31. Chang, Y.C.; Cheng, H.C.; Chiu, M.C.; Chien, Y.H. Shape Optimisation of Multi-Chamber Acoustical Plenums Using BEM, Neural Networks, and GA Method. Arch. Acoust. 2016, 41, 43-53. [CrossRef]

32. Jang, G.W.; Lee, J.W. Optimal partition layout of expansion chamber muffler with offset inlet/outlet. Int. J. Auto. Tech-Kor. 2015, 16, 885-893. [CrossRef]

33. Lee, J.W. Optimal topology of reactive muffler achieving target transmission loss values: Design and experiment. Appl. Acoust. 2015, 88, 104-113. [CrossRef]

34. Chiu, M.C. Shape optimization of multi-chamber mufflers with plug-inlet tube on a venting process by genetic algorithms. Appl. Acoust. 2010, 71, 495-505. [CrossRef]

35. Chiu, M.C. Genetic Algorithm Optimization on a Venting System with Three-Chamber Hybrid Mufflers within a Constrained Back Pressure and Space. J. Vib. Acoust. 2012, 134, 021005. [CrossRef]

36. Xu, Y.M.; Zhao, W.C.; Chen, L.L.; Chen, H.B. Distribution O-ptimization for Acoustic Design of Porous Lay-er by the Boundary Element Method. Acoust. Aust. 2020, 48, 107-119. [CrossRef]

37. Bechert, D.; Reif, W. On the Drag Reduction of the Shark Skin. In Proceedings of the 23rd Aerospace Sciences Meeting, Reno, NV, USA, 14-17 January 1985. [CrossRef]

38. Jung, Y.C.; Bhushan, B. Biomimetic Structures for Fluid Drag Reduction in Laminar and Turbulent Flows. J. Phys. Condens. Matter 2010, 22, 035104. [CrossRef]

39. Walsh, M.J. Turbulent boundary layer drag reduction using riblets. In Proceedings of the 20th Aerospace Sciences Meeting, Orlando, FL, USA, 11-14 January 1982. [CrossRef]

40. Walsh, M.J.; Anders, J.B. Riblet lebu research at Nasa Langley. Appl. Sci. Res. 1989, 46, 255-262. [CrossRef]

41. Zhan, F.L.; Xu, J.W. Virtual. Lab Acoustics Simulation Calculation from Introduction to Proficiency; Xi'an Northwest Polytechnical University Press: Xi'an, China, 2013; pp. 15-35.

42. Ffowcs Williams, J.E.; Hawkings, D.L. Sound Generated by Turbulence and Surfaces in Arbitrary Motion. Philos. Trans. R. Soc. 1969, 264, 321-342. [CrossRef]

43. Haykin, S.S. Neural Networks and Learning Machines, 3rd ed.; Pearson: New York, NY, USA, 2008; pp. 15-34.

44. Wen, Z.; Sun, H.K. MATLAB Intelligent Algorith; Tsinghua University Press: Beijing, China, 2017; pp. $24-41$.

45. Yang, Y.N.; Liu, Y.; Hu, H.T. Experimental study on noise reduction of a wavy multi-copter rotor. Appl. Acoust. 2020, 165, 107311. [CrossRef]

46. Ning, Z.; Wlezien, R.W.; Hu, H. An Experimental Study on Small UAV Propellers with Serrated Trailing Edges. In Proceedings of the 47th AIAA Fluid Dynamics Conference, Denver, CO, USA, 5-9 June 2017. [CrossRef]

47. Lee, H.M.; Lu, Z.B.; Lim, K.M.; Xie, J.L.; Lee, H.P. Quieter propeller with serrated trailing edge. Appl. Acoust. 2019, 146, 227-236. [CrossRef] 
48. Agrawal, B.R.; Sharma, A. Numerical analysis of aerodynamic noise mitigation via leading edge serrations for a rod-airfoil configuration. Int. J. Aeroacoustics 2016, 15, 734-756. [CrossRef]

49. Ji, Z.L. Acoustic Theory and Design of Silencers; Science Press: Beijing, China, 2015; p. 307. 